\title{
Smooth Wavelet Approximations of Truncated Legendre Polynomials via the Jacobi Theta Function
}

\author{
David W. Pravica, Njinasoa Randriampiry, and Michael J. Spurr
}

Mathematics Department, East Carolina University, Greenville, NC 27858, USA

Correspondence should be addressed to Michael J. Spurr; spurrm@ecu.edu

Received 7 March 2014; Accepted 14 July 2014; Published 16 October 2014

Academic Editor: Cristina Pignotti

Copyright ( 2014 David W. Pravica et al. This is an open access article distributed under the Creative Commons Attribution License, which permits unrestricted use, distribution, and reproduction in any medium, provided the original work is properly cited.

The family of $n$th order $q$-Legendre polynomials are introduced. They are shown to be obtainable from the Jacobi theta function and to satisfy recursion relations and multiplicatively advanced differential equations (MADEs) that are analogues of the recursion relations and ODEs satisfied by the $n$th degree Legendre polynomials. The $n$th order $q$-Legendre polynomials are shown to have vanishing $k$ th moments for $0 \leq k<n$, as does the $n$th degree truncated Legendre polynomial. Convergence results are obtained, approximations are given, a reciprocal symmetry is shown, and nearly orthonormal frames are constructed. Conditions are given under which a MADE remains a MADE under inverse Fourier transform. This is used to construct new wavelets as solutions of MADEs.

\section{Introduction}

The Legendre polynomials $P_{n}(\omega)$ of degree $n$ can be obtained by starting with $P_{0}(\omega)=1, P_{1}(\omega)=\omega$ and then relying on the recursive relation

$$
(n+1) P_{n+1}(\omega)=(2 n+1) \omega P_{n}(\omega)-n P_{n-1}(\omega)
$$

for $n \geq 1$ to obtain Legendre polynomials of higher degree. For instance, take $n=1$ in (1) and solve for $P_{2}(\omega)$ to find $P_{2}(\omega)=(3 / 2) \omega P_{1}(\omega)-(1 / 2) P_{0}(\omega)=(3 / 2) \omega^{2}-(1 / 2)$. Proceeding with (1), one obtains the remaining $P_{n}(\omega)$.

The $P_{n}(\omega)$ have many interesting properties. In addition to satisfying (1), the $P_{n}(\omega)$ satisfy Legendre's ordinary differential equation

$$
D_{\omega}^{2} P_{n}(\omega)=\omega^{2} D_{\omega}^{2} P_{n}(\omega)+2 \omega D_{\omega} P_{n}(\omega)-n(n+1) P_{n}(\omega),
$$

where $D_{\omega}$ denotes differentiation in the variable $\omega$; see [1]. Furthermore, the $P_{n}(\omega)$ restricted to the interval $[-1,1]$ form an orthogonal complete set for the square integrable functions $\mathscr{L}^{2}([-1,1])$ in the norm $\|\cdot\|_{2,[-1,1]}$. The $P_{n}(\omega)$ can also be constructed by a Gram-Schmidt orthogonalization on the polynomials $\left\{1, \omega, \omega^{2}, \ldots, \omega^{k}, \ldots\right\}$ on the interval $[-1,1]$ scaling so that $P_{n}(1)=1$, as required by (1). This implies the vanishing of the following moments:

$$
\int_{-1}^{1} \omega^{k} P_{n}(\omega) d \omega=0 \quad \text { for } 0 \leq k<n
$$

on [-1, 1] for $n \geq 1$; see [2]. From [3], we also have that

$$
\begin{aligned}
i^{n} \sqrt{\frac{\pi}{2}} P_{n}(\omega) \tilde{\chi}_{[-1,1]}(\omega) & =\frac{1}{\sqrt{2 \pi}} \int_{-\infty}^{\infty} e^{i \omega t} j_{n}(t) d t \\
& =\mathscr{F}^{-1}\left[j_{n}(t)\right](\omega),
\end{aligned}
$$

where $\mathscr{F}^{-1}$ denotes the inverse Fourier transform

$$
\mathscr{F}^{-1}[f(t)](\omega)=\frac{1}{\sqrt{2 \pi}} \int_{-\infty}^{\infty} e^{i \omega t} f(t) d t,
$$

where $j_{n}(t)$ denotes the $n$th spherical Bessel function of the first kind

$$
j_{n}(t) \equiv(-t)^{n}\left(\frac{1}{t} \frac{d}{d t}\right)^{n} \frac{\sin (t)}{t},
$$


where, for $\chi_{\Omega}(\omega)$ denoting the characteristic function of the set $\Omega$, we have

$$
\begin{aligned}
\tilde{\chi}_{[-1,1]}(\omega) & =\frac{1}{2}\left[\chi_{[-1,1]}(\omega)+\chi_{(-1,1)}(\omega)\right] \\
& = \begin{cases}0, & \omega \notin[-1,1], \\
1, & \omega \in(-1,1), \\
\frac{1}{2}, & \omega \in \partial[-1,1] .\end{cases}
\end{aligned}
$$

We refer to the $P_{n}(\omega) \widetilde{\chi}_{[-1,1]}(\omega)$ in (4) as the truncated $n$th degree Legendre polynomials.

In [4], the $n$th order $q$-advanced spherical Bessel functions of the first kind $j_{n}(q ; t)$ are introduced. Paralleling (6), one has that, for $q>1, n \in \mathbb{N}_{0}=\mathbb{N} \cup\{0\}$,

$$
\begin{aligned}
j_{n}(q ; t) & \equiv(-t)^{n}\left(\frac{1}{t} \frac{d}{d t}\right)^{n} \frac{{ }^{\operatorname{Sin}(t)}}{t} \\
& =(-t)^{n}\left(\frac{1}{t} \frac{d}{d t}\right)^{n} j_{0}(q ; t),
\end{aligned}
$$

where ${ }_{q} \operatorname{Sin}(t)$ is the $q$-advanced sine function

$$
q^{\operatorname{Sin}}(t) \equiv \frac{\operatorname{sign}(t) \sum_{M=-\infty}^{\infty}(-1)^{M} e^{-q^{M}|t|} q^{-M(M-1)}}{\sum_{M=-\infty}^{\infty}(-1)^{M} q^{-M^{2}}} .
$$

Since $\operatorname{Sin}(t)$ is defined to be odd, ${ }_{q} \operatorname{Sin}(t) / t$ is then even, and $(8)$ reveals $j_{n}(q ; t)$ to be even in $t$ when the order $n$ is even and odd when the order $n$ is odd. Many further interesting properties of $j_{n}(q ; t)$ and ${ }_{q} \operatorname{Sin}(t)$ and other related functions are developed in [4-6], which are good background references. For our purposes here we only note a few facts about the $j_{n}(q ; t)$. First, the $j_{n}(q ; t)$ belong to the class of Schwartz functions $\delta(\mathbb{R})$ and they are solutions to the multiplicatively advanced differential equation (MADE)

$$
j_{n}^{\prime \prime}(q ; t)+\frac{2}{t} j_{n}^{\prime}(q ; t)-\frac{n(n+1)}{t^{2}} j_{n}(q ; t)=-q^{n+3} j_{n}(q ; q t),
$$

as is proven in [4]. Note that (10) is a MADE from the fact that the argument $q t$ in the right-hand side of (10) is a multiple of $t$ by $q>1$. The inverse Fourier transforms of $\mathscr{F}^{-1}\left[j_{n}(q ; t)\right](\omega)$ are developed in [4] and given there as

$$
\begin{aligned}
\mathscr{F}^{-1}\left[j_{0}(q ; t)\right](\omega)= & \frac{-2\left(\mu_{q^{2}}\right)^{3}}{f_{0, q}(0) \sqrt{2 \pi}} \mathscr{A}_{\omega}\left(\frac{\omega}{\theta\left(q^{2} ; \omega^{2}\right)}\right), \quad(11) \\
\mathscr{F}^{-1}\left[j_{n+1}(q ; t)\right](\omega)= & i^{n+1} \\
& \times D_{\omega}^{n}\left\{\omega\left(\mathscr{A}_{\omega} \omega\right)^{n} \mathscr{F}^{-1}\left[j_{0}(q ; t)\right](\omega)\right\},
\end{aligned}
$$

where the integral operator $\mathscr{A}_{\omega}$ appearing in (11) and (12) acts on $\mathcal{S}(\mathbb{R})$ and is defined by

$$
\mathscr{A}_{\omega} f(\omega) \equiv \int_{-\infty}^{\omega} f(v) d v .
$$

In (11), one has that $\theta$ is the Jacobi theta function

$$
\begin{aligned}
\theta(\mathrm{Q} ; W) & \equiv \mu_{\mathrm{Q}} \prod_{n=0}^{\infty}\left(1+\frac{W}{\mathrm{Q}^{n}}\right)\left(1+\frac{1}{W \mathrm{Q}^{n+1}}\right) \\
& =\sum_{n=-\infty}^{\infty} \frac{W^{n}}{\mathrm{Q}^{n(n-1) / 2}}
\end{aligned}
$$

for $Q>1$, where

$$
\begin{gathered}
\mu_{\mathrm{Q}} \equiv \prod_{n=0}^{\infty}\left(1-\frac{1}{\mathrm{Q}^{n+1}}\right)>0, \\
f_{0, q}(0) \equiv \sum_{M=-\infty}^{\infty}(-1)^{M} q^{-M^{2}}=\theta\left(q^{2} ;-\frac{1}{q}\right) \\
=\mu_{q^{2}} \prod_{n \geq 0}\left(1-\frac{1}{q^{2 n+1}}\right)^{2}>0
\end{gathered}
$$

for $q>1$. From [4], one has the $q$-Wallis limit which relates $\mu_{q^{2}}$ from (15) to $f_{0, q}(0)$ from (16) asymptotically as $q \rightarrow 1^{+}$:

$$
\lim _{q \rightarrow 1^{+}} \frac{\ln (q)\left(\mu_{q^{2}}\right)^{3}}{f_{0, q}(0)}=\frac{\pi}{2} .
$$

Since most of the functions studied here will exhibit wavelet properties, we mention that function $\phi$ is considered to be a wavelet if

$$
\begin{gathered}
\phi \in \mathscr{L}^{1}(\mathbb{R}) \cap \mathscr{L}^{2}(\mathbb{R}) \cap \mathscr{L}^{\infty}(\mathbb{R}), \\
\int_{-\infty}^{\infty} \phi(t) d t=0, \quad(\text { equivalently } \mathscr{F}[\phi(t)](0)=0), \\
\int_{-\infty}^{\infty} \frac{|\mathscr{F}[\phi(t)](\omega)|^{2}}{|\omega|} d \omega<\infty .
\end{gathered}
$$

See [7] for further background on wavelets.

Solving (4) for $P_{n}(\omega) \widetilde{\chi}_{[-1,1]}(\omega)$ yields

$$
P_{n}(\omega) \tilde{\chi}_{[-1,1]}(\omega)=(-i)^{n} \sqrt{\frac{2}{\pi}} \mathscr{F}^{-1}\left[j_{n}(t)\right](\omega) .
$$

In analogy to (19), we make the following definition.

Definition 1. For $n \in \mathbb{N}_{0}$ and $q>1$, the $n$th order $q$-Legendre polynomials $\widetilde{P}_{n}(q ; \omega)$ are given by

$$
\widetilde{P}_{n}(q ; \omega) \equiv(-i)^{n} \sqrt{\frac{2}{\pi}} \mathscr{F}^{-1}\left[j_{n}(q ; t)\right](\omega) .
$$

See Figure 1 for graphical representations of $\widetilde{P}_{n}(q ; \omega)$. A main purpose of this paper is to study the functions $\widetilde{P}_{n}(q ; \omega) \in \mathcal{S}(\mathbb{R})$. These $q$-Legendre polynomials are Schwartz approximations to the truncated Legendre polynomials $P_{n}(\omega) \tilde{\chi}_{[-1,1]}(\omega) \notin \mathcal{S}(\mathbb{R})$, as the next theorem shows. 


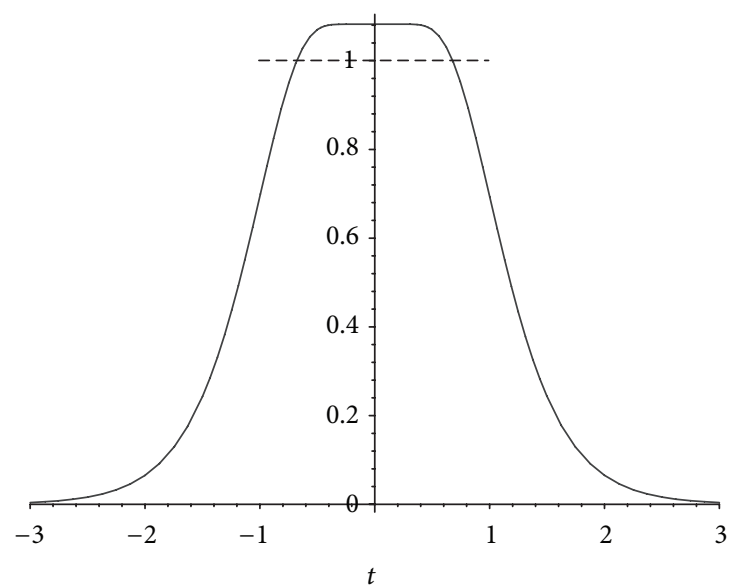

(a)

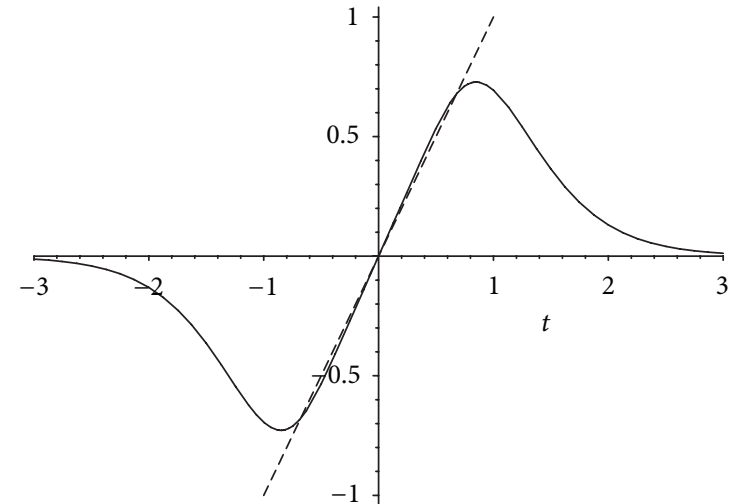

(b)

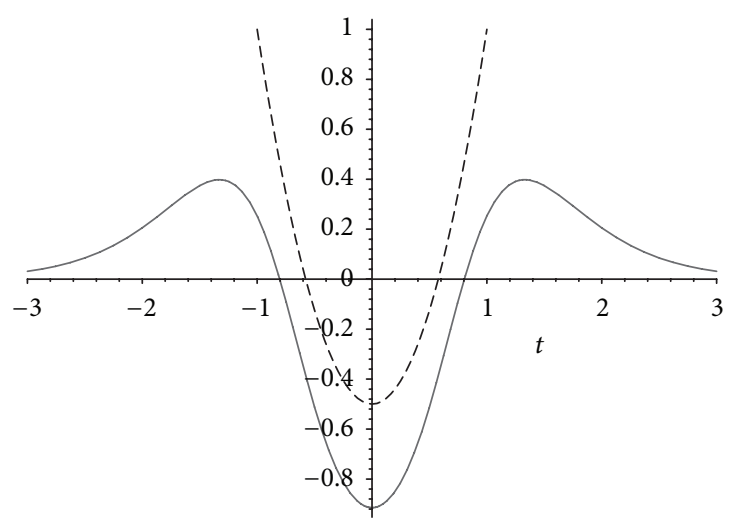

(c)

FIgURE 1: (a) $\widetilde{P}_{0}(q ; \omega)$ is shown in solid for $q=1.20$ and is compared with $P_{0}(\omega) \widetilde{\chi}_{[-1,1]}(\omega)$ which is dashed. (b) $\widetilde{P}_{1}(q ; \omega)$ is shown in solid for $q=1.20$ and is compared with $P_{1}(\omega) \widetilde{\chi}_{[-1,1]}(\omega)$ which is dashed. (c) $\widetilde{P}_{2}(q ; \omega)$ is shown in solid for $q=1.20$ and is compared with $P_{2}(\omega) \widetilde{\chi}_{[-1,1]}(\omega)$ which is dashed.

Theorem 2. The q-Legendre polynomials are Schwartz functions and are expressible in terms of the Jacobi theta function as follows:

$$
\begin{aligned}
\widetilde{P}_{0}(q ; \omega)= & \frac{-2\left(\mu_{q^{2}}\right)^{3}}{\pi f_{0, q}(0)} \mathscr{A}_{\omega}\left(\frac{\omega}{\theta\left(q^{2} ; \omega^{2}\right)}\right), \\
\widetilde{P}_{n+1}(q ; \omega)= & \frac{-2\left(\mu_{q^{2}}\right)^{3}}{\pi f_{0, q}(0)} \\
& \times D_{\omega}^{n}\left\{\left(\omega \mathscr{A}_{\omega}\right)^{n+1}\left(\frac{\omega}{\theta\left(q^{2} ; \omega^{2}\right)}\right)\right\} \quad \text { for } n \geq 0,
\end{aligned}
$$

where $\mathscr{A}_{\omega}$ is as in (13). Furthermore, for each $1 \leq p<\infty$, one has convergence in $\mathscr{L}^{p}(\mathbb{R})$ norm

$$
0=\lim _{q \rightarrow 1^{+}}\left\|\widetilde{P}_{n}(q ; \omega)-P_{n}(\omega) \tilde{\chi}_{[-1,1]}(\omega)\right\|_{p} .
$$

In addition, $\widetilde{P}_{n}(q ; \omega)$ converges pointwise to $P_{n}(\omega) \widetilde{\chi}_{[-1,1]}(\omega)$ on $\mathbb{R}$. For $n \geq 1$, the $\widetilde{P}_{n}(q ; \omega)$ are wavelets. Finally, $\widetilde{P}_{n}(q ; \omega)$ is even in $\omega$ for $n$ even and odd for $n$ odd.
Proof. To obtain the 0th order case (21), one substitutes (11) into (20). To obtain the higher order cases (22), one substitutes (12) into the $n+1$ case of (20) and then one substitutes (11) into the result to give

$$
\begin{aligned}
\widetilde{P}_{n+1}(q ; \omega)= & (-i)^{n+1} \sqrt{\frac{2}{\pi}} \mathscr{F}^{-1}\left[j_{n+1}(q ; t)\right](\omega) \\
= & (-i)^{n+1} \sqrt{\frac{2}{\pi}} i^{n+1} \\
& \times D_{\omega}^{n}\left\{\omega\left(\mathscr{A}_{\omega} \omega\right)^{n} \mathscr{F}^{-1}\left[j_{0}(q ; t)\right](\omega)\right\} \\
= & \sqrt{\frac{2}{\pi}} \frac{-2\left(\mu_{q^{2}}\right)^{3}}{f_{0, q}(0) \sqrt{2 \pi}} \\
& \times D_{\omega}^{n}\left\{\omega\left(\mathscr{A}_{\omega} \omega\right)^{n} \mathscr{A}_{\omega}\left(\frac{\omega}{\theta\left(q^{2} ; \omega^{2}\right)}\right)\right\} .
\end{aligned}
$$

Examining (20), one has that for $n \in \mathbb{N}_{0}$ the $\widetilde{P}_{n}(q ; \omega)$ are Schwartz from the fact that the $\mathscr{F}^{-1}\left[j_{n}(q ; t)\right](\omega)$ are Schwartz, which in turn follows from the fact that the 
$j_{n}(q ; t)$ are Schwartz and that $\mathscr{F}^{-1}$ preserves the Schwartz property. Similarly, for $n \in \mathbb{N}$, the fact that the $\widetilde{P}_{n}(q ; \omega)$ are wavelets follows from the fact that the $\mathscr{F}^{-1}\left[j_{n}(q ; t)\right](\omega)$ are wavelets, because the order of vanishing at $t=0$ of $\mathscr{F}\left[\mathscr{F}^{-1}\left[j_{n}(q ; t)\right]\right]=j_{n}(q ; t)$ is $n-1$ as is observed from (8) using Taylor's remainder theorem. See Theorem 8 for further discussion. The $\mathscr{L}^{p}$ convergence in (23) follows from Theorem 21 in Section 10 below. Pointwise convergence follows from Theorem 18 in Section 9 below. Finally, since the remarks following (8) give $j_{n}(q ; t)$ as even in $t$ when $n$ is even and odd when $n$ is odd, and since $\mathscr{F}^{-1}$ preserves evenness or oddness of a function, one sees from $(20)$ that $\widetilde{P}_{n}(q ; \omega)$ is even in $\omega$ when $n$ is even and odd when $n$ is odd.

Remark 3. Equation (22) in the $n+1=0$ case reduces to (21) if one interprets $D_{\omega}^{-1}=\mathscr{A}_{\omega}$. Also, Theorem 2 gives genesis to the title of this paper.

To conclude this section, we mention some useful results here. First, from $[4,6]$, the following bound holds on the reciprocal of $\theta\left(q^{2} ; \omega^{2}\right)$ :

$$
\begin{aligned}
\frac{1}{\theta\left(q^{2} ; \omega^{2}\right)} & \leq\left[q^{1 / 4}|\omega| e^{(\ln (|\omega|))^{2} / \ln (q)}\left\{\sqrt{\frac{\pi}{\ln (q)}}-1\right\}\right]^{-1} \\
& =\ln (q)\left[\frac{1}{\sqrt{\ln (q)}} \frac{q^{-1 / 4}|\omega|^{-1} e^{-(\ln (|\omega|))^{2} / \ln (q)}}{\{\sqrt{\pi}-\sqrt{\ln (q)}\}}\right]
\end{aligned}
$$

for $1<q<e^{\pi}$. This bound will be especially useful in analyzing the decay rate of the functions of interest for $\omega$ in the tails $|\omega| \geq 1+\varepsilon$.

Second, there is also a $q$-advanced cosine function

$$
{ }_{q} \operatorname{Cos}(t) \equiv \frac{\sum_{M=-\infty}^{\infty}(-1)^{M} e^{-q^{M}|t|} q^{-M^{2}}}{\sum_{M=-\infty}^{\infty}(-1)^{M} q^{-M^{2}}} .
$$

From $[4,5]$, we have the Fourier transforms

$$
\begin{aligned}
& \mathscr{F}\left[{ }_{q} \operatorname{Cos}(t)\right](\omega)=\sqrt{\frac{2}{\pi} \frac{\left(\mu_{q^{2}}\right)^{3}}{f_{0, q}(0)} \frac{1}{\theta\left(q^{2} ; \omega^{2}\right)},} \\
& \mathscr{F}\left[{ }_{q} \operatorname{Sin}(t)\right](\omega)=-i \sqrt{\frac{2}{\pi}} \frac{\left(\mu_{q^{2}}\right)^{3}}{f_{0, q}(0)} \frac{\omega}{\theta\left(q^{2} ; \omega^{2}\right)} .
\end{aligned}
$$

$\mathscr{F}\left[{ }_{q} \operatorname{Cos}(t)\right](\omega)$ will be utilized to obtain Proposition 20, which in turn helps in yielding the uniform convergence results.

\section{Main Results}

Specific properties of the $q$-Legendre polynomials $\widetilde{P}_{n}(q ; \omega)$ are established. First, we show that the $\widetilde{P}_{n}(q ; \omega)$ satisfy an analogue of the recursion relation (1), namely, (34) below.
Next, $\widetilde{P}_{n}(q ; \omega)$ also satisfy a multiplicatively advanced analogue of the Legendre ordinary differential equation (2), namely, the $q$-Legendre multiplicatively advanced differential equation (MADE) given by (56) below. Moment vanishing properties of the $\widetilde{P}_{n}(q ; \omega)$ exactly analogous to those of the truncated $P_{n}(\omega)$ in (3) are shown in (68). We obtain a reciprocal symmetry for $\widetilde{P}_{0}(q ; \omega)$ in $(71)$. The $\widetilde{P}_{n}(q ; \omega)$ are used to generate a nearly orthonormal frame for $\mathscr{L}^{2}(\mathbb{R})$ in Section 7. Alternative expressions for the $\widetilde{P}_{n}(q ; \omega)$ are obtained in Section 8. In Section 9, we obtain uniform convergence of the $\widetilde{P}_{n}(q ; \omega)$ to $P_{n}(\omega) \widetilde{\chi}_{[-1,1]}(\omega)$ as $q \rightarrow 1^{+}$on all closed sets of $\mathbb{R}$ not containing \pm 1 . This result combined with the reciprocal symmetry property then gives pointwise convergence of $\widetilde{P}_{n}(q ; \omega)$ to $P_{n}(\omega) \widetilde{\chi}_{[-1,1]}(\omega)$ on $\mathbb{R}$. In Section 10 , $\mathscr{L}^{p}$ convergence of these functions is demonstrated. Approximations to the $\widetilde{P}_{n}(q ; \omega)$ are provided in Section 11. Finally, as encountered in the process of showing the $q$-Legendre MADE, we give a more general condition under which a MADE remains a MADE under inverse Fourier transform. This is used to provide new wavelet solutions of MADEs.

It is worth mentioning that the study of MADEs and related topics has seen recent growth. See, for instance, contributions from $[4-6,8-12]$.

\section{Recursive Relations for the $\widetilde{P}_{n}(q ; \omega)$}

In this section, we obtain a $q$-version of the recursion formula (1) for $q$-Legendre polynomials, namely, (34) below. This follows from a recursion relation on the $j_{n}(q ; t)$ given by $(33)$. We begin with a lemma describing the derivative of $j_{n}(q ; t)$.

Lemma 4. For $n \in \mathbb{N}_{0}$ and $q \geq 1$, one has

$$
D_{t} j_{n}(q ; t)=\frac{n}{t} j_{n}(q ; t)-j_{n+1}(q ; t) .
$$

Proof. First, recall that $j_{0}(q ; t)={ }_{q} \operatorname{Sin}(t) / t$ and take $j_{0}(1 ; t)=$ $j_{0}(t)=\sin (t) / t$. Then,

$$
j_{n}(q ; t)=(-t)^{n}\left(\frac{1}{t} D_{t}\right)^{n} j_{0}(q ; t)
$$

is well defined. Differentiation now yields

$$
\begin{aligned}
D_{t} j_{n}(q ; t)= & -n(-t)^{n-1}\left(\frac{1}{t} D_{t}\right)^{n} j_{0}(q ; t) \\
& +(-t)^{n} D_{t}\left(\frac{1}{t} D_{t}\right)^{n} j_{0}(q ; t) \\
= & \frac{n}{t}(-t)^{n}\left(\frac{1}{t} D_{t}\right)^{n} j_{0}(q ; t) \\
& -(-t)^{n+1}\left(\frac{1}{t} D_{t}\right)^{n+1} j_{0}(q ; t) \\
= & \frac{n}{t} j_{n}(q ; t)-j_{n+1}(q ; t),
\end{aligned}
$$

giving the lemma.

Lemma 4 is the starting point in proving the following recursion relations. 
Theorem 5. For $n \in \mathbb{N}_{0}$,

$$
\begin{aligned}
(2 n+1) D_{t} j_{n}(q ; t)= & n q^{n+2} j_{n-1}(q ; q t) \\
& -(n+1) j_{n+1}(q ; t) .
\end{aligned}
$$

Or, equivalently,

$$
\begin{aligned}
(n+1) \widetilde{P}_{n+1}(q ; \omega)= & (2 n+1) \omega \widetilde{P}_{n}(q ; \omega) \\
& -n q^{n+1} \widetilde{P}_{n-1}\left(q ; \frac{\omega}{q}\right) .
\end{aligned}
$$

Proof. The $n=0$ case is handled directly. Namely, from (8), one has

$$
D_{t} j_{0}(q ; t)=-(-t)\left(\frac{1}{t} D_{t}\right) j_{0}(q ; t)=-j_{1}(q ; t),
$$

giving (33) for $n=0$. Taking inverse Fourier transforms of both sides of (35) and multiplying the resulting equation by $i \sqrt{2 / \pi}$ yield

$$
\omega \widetilde{P}_{0}(q ; \omega)=\widetilde{P}_{1}(q ; \omega)
$$

which is (34) for $n=0$. Thus, we assume $n \geq 1$ from this point on and begin first by showing (33). Setting the index $n$ equal to $n-1$ in (30) and solving the result for $j_{n}(q ; t)$ yield

$$
j_{n}(q ; t)=\frac{n-1}{t} j_{n-1}(q ; t)-D_{t} j_{n-1}(q ; t) .
$$

From (10), with the index set to be $n-1$, one obtains

$$
\begin{aligned}
D_{t}^{2} j_{n-1}(q ; t)= & \frac{n(n-1)}{t^{2}} j_{n-1}(q ; t)-\frac{2}{t} D_{t} j_{n-1}(q ; t) \\
& -q^{n+2} j_{n-1}(q ; q t) .
\end{aligned}
$$

Differentiating (37) yields

$$
\begin{aligned}
D_{t} j_{n}(q ; t)= & -\frac{(n-1)}{t^{2}} j_{n-1}(q ; t)+\frac{(n-1)}{t} D_{t} j_{n-1}(q ; t) \\
& -D_{t}^{2} j_{n-1}(q ; t) .
\end{aligned}
$$

Replacing the second derivative term in (39) with that in (38) yields

$$
\begin{aligned}
D_{t} j_{n}(q ; t)= & -\frac{(n-1)}{t^{2}} j_{n-1}(q ; t)+\frac{(n-1)}{t} D_{t} j_{n-1}(q ; t) \\
& -\frac{n(n-1)}{t^{2}} j_{n-1}(q ; t)+\frac{2}{t} D_{t} j_{n-1}(q ; t) \\
& +q^{n+2} j_{n-1}(q ; q t) \\
= & \frac{(n+1)}{t}\left[D_{t} j_{n-1}(q ; t)-\frac{(n-1)}{t} j_{n-1}(q ; t)\right] \\
& +q^{n+2} j_{n-1}(q ; q t) \\
= & \frac{(n+1)}{t}\left[-j_{n}(q ; t)\right]+q^{n+2} j_{n-1}(q ; q t)
\end{aligned}
$$

where (37) was used to replace the bracketed expression in (41) and obtain (42). Continuing yields

$$
\begin{aligned}
D_{t} j_{n}(q ; t)= & -\frac{(n+1)}{n}\left[\frac{n}{t} j_{n}(q ; t)\right]+q^{n+2} j_{n-1}(q ; q t) \\
= & -\frac{(n+1)}{n}\left[D_{t} j_{n}(q ; t)+j_{n+1}(q ; t)\right] \\
& +q^{n+2} j_{n-1}(q ; q t),
\end{aligned}
$$

where (30) was used to replace the bracketed expression in (43) and obtain (44). Multiplying (43) and (44) through by $n$ and combining terms gives (33). Note the multiplicative advance $q t$ in the argument of the $j_{n-1}$ term in (33) and (44).

To obtain (34), one takes the inverse Fourier transform of (33), relying on the fact that $\mathscr{F}^{-1}\left[D_{t} f(t)\right](\omega)=$ $(-i \omega) \mathscr{F}^{-1}[f(t)](\omega)$ to obtain

$$
\begin{aligned}
(2 n+1) & (-i \omega) \mathscr{F}^{-1}\left[j_{n}(q ; t)\right](\omega) \\
= & n q^{n+2} \mathscr{F}^{-1}\left[j_{n-1}(q ; q t)\right](\omega) \\
& -(n+1) \mathscr{F}^{-1}\left[j_{n+1}(q ; t)\right](\omega) .
\end{aligned}
$$

One next utilizes the fact that

$$
\begin{aligned}
\mathscr{F}^{-1}[f(q t)](\omega) & =\frac{1}{\sqrt{2 \pi}} \int_{-\infty}^{\infty} e^{i \omega t} f(q t) d t \\
& =\frac{1}{\sqrt{2 \pi}} \int_{-\infty}^{\infty} e^{i(\omega / q) q t} f(q t) \frac{d(q t)}{q} \\
& =\frac{1}{q} \mathscr{F}^{-1}[f(t)]\left(\frac{\omega}{q}\right)
\end{aligned}
$$

to reexpress $\mathscr{F}^{-1}\left[j_{n-1}(q ; q t)\right](\omega)$ in $(45)$, obtaining

$$
\begin{aligned}
(2 n+1) & (-i \omega) \mathscr{F}^{-1}\left[j_{n}(q ; t)\right](\omega) \\
= & n q^{n+2} q^{-1} \mathscr{F}^{-1}\left[j_{n-1}(q ; t)\right]\left(\frac{\omega}{q}\right) \\
& -(n+1) \mathscr{F}^{-1}\left[j_{n+1}(q ; t)\right](\omega) .
\end{aligned}
$$

Multiplying (47) through by $(-i)^{n-1} \sqrt{2 / \pi}$ gives

$$
\begin{aligned}
(2 n+1) \omega(-i)^{n} \sqrt{\frac{2}{\pi}} \mathscr{F}^{-1}\left[j_{n}(q ; t)\right](\omega) \\
=n q^{n+1}(-i)^{n-1} \sqrt{\frac{2}{\pi}} \mathscr{F}^{-1}\left[j_{n-1}(q ; t)\right]\left(\frac{\omega}{q}\right) \\
+(n+1)(-i)^{n+1} \sqrt{\frac{2}{\pi}} \mathscr{F}^{-1}\left[j_{n+1}(q ; t)\right](\omega) .
\end{aligned}
$$

Relying on (20) from Definition 1 gives

$$
\begin{aligned}
(2 n+1) \omega \widetilde{P}_{n}(q ; \omega)= & n q^{n+1} \widetilde{P}_{n-1}\left(q ; \frac{\omega}{q}\right) \\
& +(n+1) \widetilde{P}_{n+1}(q ; \omega) .
\end{aligned}
$$


Solving for $(n+1) \widetilde{P}_{n+1}(q ; \omega)$ gives (34) and finishes the proof. Note the multiplicative delay $\omega / q$ in the argument of the $\widetilde{P}_{n-1}$ term in (34).

As is done at the beginning of the paper for the Legendre polynomials, we utilize the new recursion relation (34) to generate the first few $q$-Legendre polynomials. Observe that $\widetilde{P}_{0}(q ; \omega)$ is given directly by (21). Next, from (22), with $n$ set to 0 , we obtain

$$
\widetilde{P}_{1}(q ; \omega)=\omega \widetilde{P}_{0}(q ; \omega) .
$$

From (34), with $n=1$, one obtains

$$
\begin{aligned}
\widetilde{P}_{2}(q ; \omega) & =\frac{3}{2} \omega \widetilde{P}_{1}(q ; \omega)-\frac{1}{2} q^{2} \widetilde{P}_{0}\left(q ; \frac{\omega}{q}\right) \\
& =\frac{3}{2} \omega^{2} \widetilde{P}_{0}(q ; \omega)-\frac{1}{2} q^{2} \widetilde{P}_{0}\left(q ; \frac{\omega}{q}\right) .
\end{aligned}
$$

From (34), with $n=2$, one obtains

$$
\begin{aligned}
\widetilde{P}_{3}(q ; \omega)= & \frac{5}{3} \omega \widetilde{P}_{2}(q ; \omega)-\frac{2}{3} q^{3} \widetilde{P}_{1}\left(q ; \frac{\omega}{q}\right) \\
= & \frac{5}{3} \omega\left\{\frac{3}{2} \omega^{2} \widetilde{P}_{0}(q ; \omega)-\frac{1}{2} q^{2} \widetilde{P}_{0}\left(q ; \frac{\omega}{q}\right)\right\} \\
& -\frac{2}{3} q^{3}\left\{\frac{\omega}{q \widetilde{P}_{0}(q ; \omega / q)}\right\} \\
= & \frac{5}{2} \omega^{3} \widetilde{P}_{0}(q ; \omega)-\frac{3}{2} q^{2} \omega \widetilde{P}_{0}\left(q ; \frac{\omega}{q}\right) .
\end{aligned}
$$

From (34), with $n=3$, one obtains

$$
\begin{aligned}
\widetilde{P}_{4}(q ; \omega)= & \frac{7}{4} \omega \widetilde{P}_{3}(q ; \omega)-\frac{3}{4} q^{4} \widetilde{P}_{2}\left(q ; \frac{\omega}{q}\right) \\
= & \frac{7}{4} \omega\left\{\frac{5}{2} \omega^{3} \widetilde{P}_{0}(q ; \omega)-\frac{3}{2} q^{2} \omega \widetilde{P}_{0}\left(q ; \frac{\omega}{q}\right)\right\} \\
& -\frac{3}{4} q^{4}\left\{\frac{3}{2}\left(\frac{\omega}{q}\right)^{2} \widetilde{P}_{0}\left(q ; \frac{\omega}{q}\right)-\frac{1}{2} q^{2} \widetilde{P}_{0}\left(q ; \frac{\omega}{q^{2}}\right)\right\} \\
= & \frac{35}{8} \omega^{4} \widetilde{P}_{0}(q ; \omega)-\frac{21}{8} q^{2} \omega^{2} \widetilde{P}_{0}\left(q ; \frac{\omega}{q}\right) \\
& -\frac{9}{8} q^{2} \omega^{2} \widetilde{P}_{0}\left(q ; \frac{\omega}{q}\right)+\frac{3}{8} q^{6} \widetilde{P}_{0}\left(q ; \frac{\omega}{q^{2}}\right) \\
= & \frac{35}{8} \omega^{4} \widetilde{P}_{0}(q ; \omega)-\frac{30}{8} q^{2} \omega^{2} \widetilde{P}_{0}\left(q ; \frac{\omega}{q}\right) \\
& +\frac{3}{8} q^{6} \widetilde{P}_{0}\left(q ; \frac{\omega}{q^{2}}\right) .
\end{aligned}
$$

Proceeding on, one obtains the general $n$th order $q$-Legendre polynomial by multiplying each term of the $n$th degree Legendre polynomial by a power of $q$ and by a multiplicative delay of $\widetilde{P}_{0}(q ; \omega)$ by a power of $q$ and then summing. The expression extending (50)-(55) to general $n$ will be given in Theorem 11 and Corollary 13 in Section 8 below.

\section{MADEs for the $\widetilde{P}_{n}(q ; \omega)$}

In this section the $q$-Legendre polynomials are shown to satisfy a $q$-version of Legendre's ODE (2), namely, the multiplicatively advanced differential equation (MADE) given by (56) below.

Theorem 6. The q-Legendre polynomial $\widetilde{P}_{n}(q ; \omega)=$ $(-i)^{n} \sqrt{2 / \pi} \mathscr{F}^{-1}\left[j_{n}(q ; t)\right](\omega)$ satisfies the multiplicatively advanced differential equation

$$
\begin{aligned}
{\left[D_{\omega}^{2} \widetilde{P}_{n}\right](q ; \omega)=} & q^{-n}(q \omega)^{2}\left[D_{\omega}^{2} \widetilde{P}_{n}\right](q ; q \omega) \\
& +2 q^{-n}(q \omega)\left[D_{\omega} \widetilde{P}_{n}\right](q ; q \omega) \\
& -q^{-n} n(n+1) \widetilde{P}_{n}(q ; q \omega)
\end{aligned}
$$

This reduces to the Legendre differential equation (2) as $q \rightarrow$ $1^{+}$with $\widetilde{P}_{n}(1 ; \omega) \equiv P_{n}(\omega) \widetilde{\chi}_{[-1,1]}(\omega)$ for $|\omega|<1$.

Proof. Multiplying (10) by $t^{2}$ yields

$$
\begin{aligned}
& t^{2} j_{n}^{\prime \prime}(q ; t)+2 t j_{n}^{\prime}(q ; t)-n(n+1) j_{n}(q ; t) \\
& =-q^{n+3} t^{2} j_{n}(q ; q t) .
\end{aligned}
$$

Relying on the facts that

$$
\begin{aligned}
& \mathscr{F}^{-1}[t f(t)](\omega)=\left(-i D_{\omega}\right) \mathscr{F}^{-1}[f(t)](\omega), \\
& \mathscr{F}^{-1}\left[D_{t} f(t)\right](\omega)=(-i \omega) \mathscr{F}^{-1}[f(t)](\omega),
\end{aligned}
$$

one applies the inverse Fourier transform $\mathscr{F}^{-1}$ to $(57)$ to obtain

$$
\begin{aligned}
\left(-i D_{\omega}\right)^{2}(-i \omega)^{2} \mathscr{F}^{-1}\left[j_{n}(q ; t)\right](\omega) \\
\quad+2\left(-i D_{\omega}\right)(-i \omega) \mathscr{F}^{-1}\left[j_{n}(q ; t)\right](\omega) \\
\quad-n(n+1) \mathscr{F}^{-1}\left[j_{n}(q ; t)\right](\omega) \\
=-q^{n+3}\left(-i D_{\omega}\right)^{2} \mathscr{F}^{-1}\left[j_{n}(q ; q t)\right](\omega) .
\end{aligned}
$$

Simplifying the left-hand side of (59) and relying on (46) to simplify the right-hand side of (59) yields

$$
\begin{aligned}
& D_{\omega}^{2} \omega^{2} \mathscr{F}^{-1}\left[j_{n}(q ; t)\right](\omega)-2 D_{\omega} \omega \mathscr{F}^{-1}\left[j_{n}(q ; t)\right](\omega) \\
&-n(n+1) \mathscr{F}^{-1}\left[j_{n}(q ; t)\right](\omega) \\
&= q^{n+3} D_{\omega}^{2}\left\{\frac{1}{q} \mathscr{F}^{-1}\left[j_{n}(q ; t)\right]\left(\frac{\omega}{q}\right)\right\} .
\end{aligned}
$$


Using the derivation property of $D_{\omega}$ on the left-hand side of (61) gives

$$
\begin{aligned}
2 \mathscr{F}^{-1} & {\left[j_{n}(q ; t)\right](\omega)+4 \omega D_{\omega} \mathscr{F}^{-1}\left[j_{n}(q ; t)\right](\omega) } \\
& +\omega^{2} D_{\omega}^{2} \mathscr{F}^{-1}\left[j_{n}(q ; t)\right](\omega) \\
& -2 \mathscr{F}^{-1}\left[j_{n}(q ; t)\right](\omega) \\
& -2 \omega D_{\omega} \mathscr{F}^{-1}\left[j_{n}(q ; t)\right](\omega) \\
& -n(n+1) \mathscr{F}^{-1}\left[j_{n}(q ; t)\right](\omega) \\
= & q^{n+2} D_{\omega}^{2}\left\{\mathscr{F}^{-1}\left[j_{n}(q ; t)\right]\left(\frac{\omega}{q}\right)\right\} .
\end{aligned}
$$

Simplifying (62)-(63) and multiplying through by $(-i)^{n} \sqrt{2 / \pi}$ yield

$$
\begin{aligned}
& \omega^{2} D_{\omega}^{2} \widetilde{P}_{n}(q ; \omega)+2 \omega D_{\omega} \widetilde{P}_{n}(q ; \omega)-n(n+1) \widetilde{P}_{n}(q ; \omega) \\
& =q^{n+2} D_{\omega}^{2}\left\{\widetilde{P}_{n}\left(q ; \frac{\omega}{q}\right)\right\} .
\end{aligned}
$$

Letting $u=\omega / q$ with $D_{\omega}=(1 / q) D_{u}$ yields

$$
\begin{aligned}
(q u)^{2} & \left\{\frac{1}{q} D_{u}\right\}^{2}\left\{\widetilde{P}_{n}(q ; q u)\right\} \\
& +2(q u)\left\{\frac{1}{q} D_{u}\right\}\left\{\widetilde{P}_{n}(q ; q u)\right\}-n(n+1) \widetilde{P}_{n}(q ; q u) \\
= & q^{n+2}\left\{\frac{1}{q} D_{u}\right\}^{2} \widetilde{P}_{n}(q ; u) .
\end{aligned}
$$

Thus, we solve for the right-hand side of (65) scaled by $q^{-n}$ to obtain

$$
\begin{aligned}
D_{u}^{2} \widetilde{P}_{n}(q ; u)= & q^{-n}(q u)^{2}\left\{\frac{1}{q} D_{u}\right\}^{2}\left\{\widetilde{P}_{n}(q ; q u)\right\} \\
& +2 q^{-n}(q u)\left\{\frac{1}{q} D_{u}\right\}\left\{\widetilde{P}_{n}(q ; q u)\right\} \\
& -q^{-n} n(n+1) \widetilde{P}_{n}(q ; q u),
\end{aligned}
$$

which simplifies to (56) after a final substitution $u=\omega$. The theorem is now proven.

Remark 7. Equation (56) is appropriately considered to be a MADE over the apparent delayed differential equation (64) in that the term with the highest order derivative with constant coefficient should be the dominant term for small $|\omega|$ and thus expressed in terms of the unscaled variable. This will be further addressed in Section 12.

\section{Vanishing of Moments for the $\widetilde{P}_{n}(q ; \omega)$}

Let $n \geq 1$. In light of (19), (3) can be rewritten as

$$
\begin{aligned}
0 & =\int_{-\infty}^{\infty} \omega^{k} \tilde{\chi}_{[-1,1]}(\omega) P_{n}(\omega) d \omega \\
& =\int_{-\infty}^{\infty} \omega^{k}(-i)^{n} \sqrt{\frac{2}{\pi}} \mathscr{F}^{-1}\left[j_{n}(t)\right](\omega) d \omega \quad \text { for } 0 \leq k<n,
\end{aligned}
$$

which tells us that the 0th through $(n-1)$ th moments of the truncated $n$th degree Legendre polynomial vanish. In light of (20) in Definition 1, the statement analogous to (67) is given by (68) in the next theorem.

Theorem 8. Let $n \geq 1$. The 0th through $(n-1)$ th moments of the nth order q-Legendre polynomial vanish. Consider

$$
\begin{aligned}
0 & =\int_{-\infty}^{\infty} \omega^{k} \widetilde{P}_{n}(q ; \omega) d \omega \\
& =\int_{-\infty}^{\infty} \omega^{k}(-i)^{n} \sqrt{\frac{2}{\pi}} \mathscr{F}^{-1}\left[j_{n}(q ; t)\right](\omega) d \omega \quad \text { for } 0 \leq k<n .
\end{aligned}
$$

The proof is outlined here. Recall that the $k$ th moment of $f$ vanishing is equivalent to the $k$ th derivative of $\mathscr{F}[f]$ vanishing at 0 . From (20), one has immediately that, for all $n \in \mathbb{N}$,

$$
\begin{aligned}
\mathscr{F}\left[\widetilde{P}_{n}(q ; \omega)\right](t) & =(-i)^{n} \sqrt{\frac{2}{\pi}} \mathscr{F}\left[\mathscr{F}^{-1}\left[j_{n}(q ; t)\right](\omega)\right](t) \\
& =(-i)^{n} \sqrt{\frac{2}{\pi}} j_{n}(q ; t) \\
& =(-i)^{n} \sqrt{\frac{2}{\pi}}(-t)^{n}\left(\frac{1}{t} \frac{d}{d t}\right)^{n} j_{0}(q ; t),
\end{aligned}
$$

where the last equality follows from (8). Now, the $(-t)^{n}$ factor and the outer $1 / t$ factor in (69) guarantee that the first $n-1$ derivatives of $\mathscr{F}\left[\widetilde{P}_{n}(q ; \omega)\right](t)$ vanish at $t=0$, after noting that the derivatives of $((1 / t)(d / d t))^{n} j_{0}(q ; t)$ are bounded for all $n \epsilon$ $\mathbb{N}_{0}$. This gives (68). In contrast, it is shown in [4] that the $n$th derivative of $\mathscr{F}\left[\widetilde{P}_{n}(q ; \omega)\right](t)$ does not vanish at $t=0$.

\section{A Reciprocal Symmetry for $\widetilde{P}_{0}(q ; \omega)$}

There is an interesting reciprocal symmetry satisfied by $\widetilde{P}_{0}(q ; \omega)$, and this will help produce a pointwise convergence result in Section 9.

Theorem 9. For all $\omega \in \mathbb{R}$. one has

$$
\widetilde{P}_{0}(q ; 0)-\widetilde{P}_{0}(q ; \omega)=\widetilde{P}_{0}\left(q ; \frac{q}{\omega}\right),
$$

or equivalently

$$
\widetilde{P}_{0}(q ; 0)-\widetilde{P}_{0}(q ; \sqrt{q} \omega)=\widetilde{P}_{0}\left(q ; \frac{\sqrt{q}}{\omega}\right) .
$$


Proof. From (21), we have, for $\omega<0$,

$$
\begin{aligned}
& \widetilde{P}_{0}(q ; 0)-\widetilde{P}_{0}(q ; \omega)=\frac{-2\left(\mu_{q^{2}}\right)^{3}}{\pi f_{0, q}(0)} \int_{-\infty}^{0} \frac{v}{\theta\left(q^{2} ; v^{2}\right)} d v \\
& -\frac{-2\left(\mu_{q^{2}}\right)^{3}}{\pi f_{0, q}(0)} \int_{-\infty}^{\omega} \frac{v}{\theta\left(q^{2} ; v^{2}\right)} d v \\
& =\frac{-2\left(\mu_{q^{2}}\right)^{3}}{\pi f_{0, q}(0)} \int_{\omega}^{0} \frac{v}{\theta\left(q^{2} ; v^{2}\right)} d v \\
& =\frac{-2\left(\mu_{q^{2}}\right)^{3}}{\pi f_{0, q}(0)} \int_{1 / \omega}^{-\infty} \frac{u^{-1}}{\theta\left(q^{2} ; u^{-2}\right)} \frac{-d u}{u^{2}} \\
& =\frac{-2\left(\mu_{q^{2}}\right)^{3}}{\pi f_{0, q}(0)} \int_{-\infty}^{1 / \omega} \frac{u^{-1}}{\theta\left(q^{2} ; u^{-2}\right)} \frac{d u}{u^{2}} \\
& =\frac{-2\left(\mu_{q^{2}}\right)^{3}}{\pi f_{0, q}(0)} \int_{-\infty}^{1 / \omega} \frac{u^{-1}}{\theta\left(q^{2} ; u^{2}\right)} d u \\
& =\frac{-2\left(\mu_{q^{2}}\right)^{3}}{\pi f_{0, q}(0)} \int_{-\infty}^{1 / \omega} \frac{q^{2} u}{q^{2} u^{2} \theta\left(q^{2} ; u^{2}\right)} d u \\
& =\frac{-2\left(\mu_{q^{2}}\right)^{3}}{\pi f_{0, q}(0)} \int_{-\infty}^{1 / \omega} \frac{q u}{\theta\left(q^{2} ;(q u)^{2}\right)} d(q u) \\
& =\frac{-2\left(\mu_{q^{2}}\right)^{3}}{\pi f_{0, q}(0)} \int_{-\infty}^{q / \omega} \frac{v}{\theta\left(q^{2} ; v^{2}\right)} d v \\
& =\widetilde{P}_{0}\left(q ; \frac{q}{\omega}\right) \text {. }
\end{aligned}
$$

Here, the change of variables $u=1 / v$ was used to obtain (74), the identity $u^{2} \theta\left(q^{2} ; u^{-2}\right)=\theta\left(q^{2} ; u^{2}\right)$ was used to obtain (76), the integrand in (76) was multiplied by $q^{2} u^{2} /\left(q^{2} u^{2}\right)$ to obtain (77), the identity $q^{2} u^{2} \theta\left(q^{2} ; u^{2}\right)=\theta\left(q^{2} ; q^{2} u^{2}\right)$ was used to obtain (78), the change of variables $v=q u$ was used to obtain (79), and (21) was used to obtain (80) above. Thus, (70) holds for $\omega<0$. By replacing $\omega$ by $\sqrt{q} \omega$ in the expression (70), one obtains (71) for $\omega<0$. Note that, by evenness of $\widetilde{P}_{0}(q ; \omega)$, one has that both the identities (70) and (71) hold for all $\omega \in \mathbb{R}$.

\section{Nearly Orthonormal Frames from the $\widetilde{P}_{n}(q ; \omega)$}

As in [13], a countable set of functions $\left\{g_{k}(t) \mid k \in \mathbb{N}\right\} \subset$ $\mathscr{L}^{2}(\mathbb{R})$ is a frame for $\mathscr{L}^{2}(\mathbb{R})$ if there are constants $0<A \leq$ $B<\infty$ with

$$
A\|f\|_{2}^{2} \leq \sum_{k \in \mathbb{N}}\left|\left\langle f(t), g_{k}(t)\right\rangle\right|^{2} \leq B\|f\|_{2}^{2} \quad \forall f \in \mathscr{L}^{2}(\mathbb{R}) .
$$

The frame condition (81) is equivalent to

$$
A \leq \sum_{k \in \mathbb{N}}\left|\left\langle\frac{f(t)}{\|f\|_{2}}, g_{k}(t)\right\rangle\right|^{2} \leq B \quad \forall f \in \mathscr{L}^{2}(\mathbb{R}) \backslash 0 .
$$

We construct a frame from the $\widetilde{P}_{n}(q ; \omega)$ in the following manner. For each $n \in \mathbb{N}_{0}=\mathbb{N} \cup\{0\}$ and $k \in \mathbb{Z}$, let

$$
\begin{aligned}
w_{n, k}(\omega) & \equiv C_{n} P_{n}(\omega-2 k) \tilde{\chi}_{[-1,1]}(\omega-2 k), \\
\widehat{w}_{n, k, q}(\omega) & \equiv C_{n} \widetilde{P}_{n}(q ; \omega-2 k),
\end{aligned}
$$

where $C_{n}=\sqrt{1 /(n+1 / 2)}$ normalizes $w_{n, k}$ to give $\left\|w_{n, k}\right\|_{2}=1$, see [1].

From (23), it follows that

$$
\lim _{q \rightarrow 1^{+}}\left\|\widehat{w}_{n, k, q}(\omega)-w_{n, k}(\omega)\right\|_{2}=0
$$

Thus, for each $1>\varepsilon>0$, there is a $q_{(n, k, \varepsilon)}>1$ such that for all $q$ with $1<q \leq q_{(n, k, \varepsilon)}$

$$
\left\|\widehat{w}_{n, k, q}(\omega)-w_{n, k}(\omega)\right\|_{2}<\frac{\varepsilon}{18 \cdot 2^{n} \cdot 2^{|k|}} .
$$

For conciseness, by suppressing $\varepsilon$ and $q_{(n, k, \varepsilon)}$, set

$$
\widetilde{w}_{n, k}(\omega) \equiv \widehat{w}_{n, k, q_{(n, k, \varepsilon)}}(\omega)
$$

Theorem 10. Given $\varepsilon \in(0,1)$, the associated set of functions $\left\{\widetilde{w}_{n, k}(\omega) \mid n \in \mathbb{N}_{0}, k \in \mathbb{Z}\right\}$, with $\widetilde{w}_{n, k}(\omega)$, as in (87), is a nearly orthonormal frame for $\mathscr{L}^{2}(\mathbb{R})$ in the sense that

$$
\left|\left\langle\widetilde{w}_{N, K}, \widetilde{w}_{n, k}\right\rangle-\delta_{N}^{n} \cdot \delta_{K}^{k}\right|<\varepsilon
$$

where the Kronecker delta function $\delta_{k}^{j}$ satisfies $\delta_{k}^{j}=0$ for $k \neq j$ and $\delta_{j}^{j}=1$.

Proof. We start by noting that

$$
\sum_{n \in \mathbb{N}_{0}} \sum_{k \in \mathbb{Z}}\left|\left\langle\frac{f(t)}{\|f\|_{2}}, w_{n, k}(t)\right\rangle\right|^{2}=1 \quad \forall f \in \mathscr{L}^{2}(\mathbb{R}) \backslash 0 .
$$

This follows since the $w_{n, 0}$ are orthonormal and complete on $[-1,1]$, as in [2]. Thus the translates of $w_{n, 0}$ by multiples 2 , namely the $w_{n, k}$, are orthonormal and complete in $\mathscr{L}^{2}(\mathbb{R})$. Let $1>\varepsilon>0$ be given. We bootstrap on the fact that $\left\{w_{n, k}\right\}$ is an orthonormal frame to show that smooth approximations 
$\left\{\widetilde{w}_{n, k}\right\}$ given by $(87)$ are also a frame for $\mathscr{L}^{2}(\mathbb{R})$. For all functions $f \in \mathscr{L}^{2}(\mathbb{R}) \backslash\{0\}$, one computes that

$$
\begin{aligned}
& \sum_{n \in \mathbb{N}_{0}} \sum_{k \in \mathbb{Z}}\left|\left\langle\frac{f(t)}{\|f\|_{2}}, \widetilde{w}_{n, k}(t)\right\rangle\right|^{2} \\
&=\sum_{n \in \mathbb{N}_{0}} \sum_{k \in \mathbb{Z}} \mid\left\langle\frac{f(t)}{\|f\|_{2}}, w_{n, k}(t)\right\rangle \\
&+\left.\left\langle\frac{f(t)}{\|f\|_{2}}, \widetilde{w}_{n, k}(t)-w_{n, k}(t)\right\rangle\right|^{2} \\
&=\sum_{n \in \mathbb{N}_{0}} \sum_{k \in \mathbb{Z}}\left\{\left|\left\langle\frac{f(t)}{\|f\|_{2}}, w_{n, k}(t)\right\rangle\right|^{2}\right. \\
&+2 \Re\left\{\left\langle\frac{f(t)}{\|f\|_{2}}, w_{n, k}(t)\right\rangle\right. \\
&+\sum_{n \in \mathbb{N}_{0}} \sum_{k \in \mathbb{Z}} \mid\left\langle\frac{f(t)}{\|f\|_{2}}, \widetilde{w}_{n, k}(t)-w_{n, k}(t)\right\rangle \\
&\left.+\left|\left\langle\frac{f(t)}{\|f\|_{2}}, \widetilde{w}_{n, k}(t)-w_{n, k}(t)\right\rangle\right|^{2}\right\} \\
&\left.\times 1+\sum_{n \in \mathbb{N}_{0}} \sum_{k \in \mathbb{Z}} 2 \Re\left\{\frac{\left.f(t)-w_{n, k}(t)\right\rangle\left.\right|^{2}}{\|f\|_{2}}, \widetilde{w}_{n, k}(t)-w_{n, k}(t)\right\rangle\right\} \\
&\left(9 f \|_{2}, w_{n, k}(t)\right\rangle
\end{aligned}
$$

Now, one uses Cauchy-Schwarz and (86) to obtain the bound

$$
\begin{aligned}
& \left|2 \Re\left\{\left\langle\frac{f(t)}{\|f\|_{2}}, w_{n, k}(t)\right\rangle \overline{\left\langle\frac{f(t)}{\|f\|_{2}}, \widetilde{w}_{n, k}(t)-w_{n, k}(t)\right\rangle}\right\}\right| \\
& \leq 2\left|\left\langle\frac{f(t)}{\|f\|_{2}}, w_{n, k}(t)\right\rangle\left\langle\frac{f(t)}{\|f\|_{2}}, \widetilde{w}_{n, k}(t)-w_{n, k}(t)\right\rangle\right| \\
& \leq 2\left\|\frac{f(t)}{\|f\|_{2}}\right\|_{2}\left\|w_{n, k}(t)\right\|_{2}\left\|\frac{f(t)}{\|f\|_{2}}\right\|_{2}\left\|\widetilde{w}_{n, k}(t)-w_{n, k}(t)\right\|_{2} \\
& =2 \cdot 1 \cdot 1 \cdot 1\left\|\widetilde{w}_{n, k}(t)-w_{n, k}(t)\right\|_{2} \leq 2 \frac{\varepsilon}{18 \cdot 2^{n} \cdot 2^{|k|}} .
\end{aligned}
$$

Thus, one can bound (90) from below by discarding the last term of (92) and relying on (94) to obtain (96) as follows:

$$
\begin{aligned}
& \sum_{n \in \mathbb{N}_{0}} \sum_{k \in \mathbb{Z}}\left|\left\langle\frac{f(t)}{\|f\|_{2}}, \widetilde{w}_{n, k}(t)\right\rangle\right|^{2} \\
& \geq 1+\sum_{n \in \mathbb{N}_{0}} \sum_{k \in \mathbb{Z}} 2 \Re\left\{\left\langle\frac{f(t)}{\|f\|_{2}}, w_{n, k}(t)\right\rangle\right. \\
& \left.\times \overline{\left\langle\frac{f(t)}{\|f\|_{2}}, \widetilde{w}_{n, k}(t)-w_{n, k}(t)\right\rangle}\right\} \\
& \geq 1-\sum_{n \in \mathbb{N}_{0}} \sum_{k \in \mathbb{Z}} 2 \frac{\varepsilon}{18 \cdot 2^{n} \cdot 2^{|k|}} \\
& =1-\frac{2 \varepsilon}{18} \sum_{n=0}^{\infty} \frac{1}{2^{n}}\left\{\sum_{k=0}^{\infty} \frac{1}{2^{|k|}}+\sum_{k=-\infty}^{-1} \frac{1}{2^{|k|}}\right\} \\
& =1-\frac{2 \varepsilon}{18} 2\{2+1\}=1-\frac{12 \varepsilon}{18} \\
& \geq 1-\varepsilon \text {. }
\end{aligned}
$$

Similarly, one bounds (92) from the above by bounding the last two terms in (92) via (94) as follows:

$$
\begin{aligned}
\sum_{n \in \mathbb{N}_{0}} \sum_{k \in \mathbb{Z}}\left|\left\langle\frac{f(t)}{\|f\|_{2}}, \widetilde{w}_{n, k}(t)\right\rangle\right|^{2} \\
\leq 1+\sum_{n \in \mathbb{N}_{0}} \sum_{k \in \mathbb{Z}} 2 \frac{\varepsilon}{18 \cdot 2^{n} \cdot 2^{|k|}} \\
+\sum_{n \in \mathbb{N}_{0}} \sum_{k \in \mathbb{Z}}\left(\frac{\varepsilon}{18 \cdot 2^{n} \cdot 2^{|k|}}\right)^{2} \\
\leq 1+\sum_{n \in \mathbb{N}_{0}} \sum_{k \in \mathbb{Z}} 2 \frac{\varepsilon}{18 \cdot 2^{n} \cdot 2^{|k|}} \\
+\sum_{n \in \mathbb{N}_{0}} \sum_{k \in \mathbb{Z}} 1 \cdot\left(\frac{\varepsilon}{18 \cdot 2^{n} \cdot 2^{|k|}}\right) \\
=1+\frac{3 \varepsilon}{18} \sum_{n=0}^{\infty} \frac{1}{2^{n}}\left\{\sum_{k=0}^{\infty} \frac{1}{2^{|k|}}+\sum_{k=-\infty}^{-1} \frac{1}{2^{|k|}}\right\} \\
=1+\frac{3 \varepsilon}{18} 2\{2+1\}=1+\frac{18 \varepsilon}{18} \\
\leq 1+\varepsilon .
\end{aligned}
$$

Thus, combining (98) with (100) yields that, for all functions $f \in \mathscr{L}^{2}(\mathbb{R}) \backslash\{0\}$,

$$
1-\varepsilon \leq \sum_{n \in \mathbb{N}_{0}} \sum_{k \in \mathbb{Z}}\left|\left\langle\frac{f(t)}{\|f\|_{2}}, \widetilde{w}_{n, k}(t)\right\rangle\right|^{2} \leq 1+\varepsilon .
$$

Hence, given $\varepsilon<1$, the associated set $\left\{\widetilde{w}_{n, k}\right\}$ is a frame for $\mathscr{L}^{2}(\mathbb{R})$. 
Next, we show near orthonormality of the frame $\left\{\widetilde{w}_{n, k}\right\}$. Observe that, given $\varepsilon<1$, one has

$$
\begin{aligned}
& \left|\left\langle\widetilde{w}_{N, K}, \widetilde{w}_{n, k}\right\rangle-\left\langle w_{N, K}, w_{n, k}\right\rangle\right| \\
& =\left|\left\langle\widetilde{w}_{N, K}-w_{N, K}, \widetilde{w}_{n, k}\right\rangle+\left\langle w_{N, K}, \widetilde{w}_{n, k}-w_{n, k}\right\rangle\right| \\
& \leq\left|\left\langle\widetilde{w}_{N, K}-w_{N, K}, \widetilde{w}_{n, k}\right\rangle\right|+\left|\left\langle w_{N, K}, \widetilde{w}_{n, k}-w_{n, k}\right\rangle\right| \\
& \leq\left\|\widetilde{w}_{N, K}-w_{N, K}\right\|_{2}\left\|\widetilde{w}_{n, k}\right\|_{2}+\left\|w_{N, K}\right\|_{2}\left\|\widetilde{w}_{n, k}-w_{n, k}\right\|_{2} \\
& =\left\|\widetilde{w}_{N, K}-w_{N, K}\right\|_{2}\left\|w_{n, k}+\widetilde{w}_{n, k}-w_{n, k}\right\|_{2} \\
& +1 \cdot\left\|\widetilde{w}_{n, k}-w_{n, k}\right\|_{2} \\
& \leq\left\|\widetilde{w}_{N, K}-w_{N, K}\right\|_{2}\left\{\left\|w_{n, k}\right\|_{2}+\left\|\widetilde{w}_{n, k}-w_{n, k}\right\|_{2}\right\} \\
& +\left\|\widetilde{w}_{n, k}-w_{n, k}\right\|_{2} \\
& \leq \frac{\varepsilon}{18 \cdot 2^{N} \cdot 2^{|K|}}\left\{1+\frac{\varepsilon}{18 \cdot 2^{n} \cdot 2^{|k|}}\right\}+\frac{\varepsilon}{18 \cdot 2^{n} \cdot 2^{|k|}} \\
& \leq \frac{\varepsilon}{18}\left\{1+\frac{1}{18}\right\}+\frac{\varepsilon}{18}<\varepsilon \text {. }
\end{aligned}
$$

Thus,

$$
\begin{aligned}
\left|\left\langle\widetilde{w}_{N, K}, \widetilde{w}_{n, k}\right\rangle-\left\langle w_{N, K}, w_{n, k}\right\rangle\right| & =\left|\left\langle\widetilde{w}_{N, K}, \widetilde{w}_{n, k}\right\rangle-\delta_{N}^{n} \cdot \delta_{K}^{k}\right| \\
& <\varepsilon,
\end{aligned}
$$

where $\delta_{N}^{n}$ is the Kronecker delta function. We conclude that $\left\{\widetilde{w}_{n, k}\right\}$ is a nearly orthonormal frame.

\section{Alternative Expressions for $\widetilde{P}_{n}(q ; \omega)$}

The goal of this section is to provide alternative expressions for $\widetilde{P}_{n}(q ; \omega)$ that extend equations $(50)-(55)$. This will be done in Theorem 11 and Corollary 13 below. We obtain this extension by consulting [2] and expressing the $n$th degree Legendre polynomial as

$$
P_{n}(\omega)=\sum_{k=0}^{\lfloor n / 2\rfloor} C_{n, n-2 k} \omega^{n-2 k}
$$

where

$$
C_{n, n-2 k}=(-1)^{k} \frac{(2 n-2 k) !}{2^{n} k !(n-k) !(n-2 k) !}
$$

and $\lfloor x\rfloor$ denotes the greatest integer function. For $n \geq 1$, the recursion relation (1) in this notation takes the form

$$
\begin{aligned}
\sum_{k=0}^{\lfloor(n+1) / 2\rfloor} C_{n+1, n+1-2 k} \omega^{n+1-2 k} & \\
= & \sum_{k=0}^{\lfloor n / 2\rfloor} \frac{(2 n+1)}{(n+1)} C_{n, n-2 k} \omega^{n+1-2 k} \\
& -\sum_{k=0}^{\lfloor(n-1) / 2\rfloor} \frac{n}{(n+1)} C_{n-1, n-1-2 k} \omega^{n-1-2 k}
\end{aligned}
$$

$$
\begin{aligned}
= & \sum_{k=0}^{\lfloor n / 2\rfloor} \frac{(2 n+1)}{(n+1)} C_{n, n-2 k} \omega^{n+1-2 k} \\
& -\sum_{k=1}^{\lfloor(n-1) / 2\rfloor+1} \frac{n}{(n+1)} C_{n-1, n+1-2 k} \omega^{n+1-2 k}
\end{aligned}
$$

after reindexing $k$ in the rightmost summation in (107) to obtain (108). This implies a recursion relation on the coefficients of like powers of $\omega$ obtained in setting (106) equal to (108).

We are now prepared to state the next theorem generalizing (50)-(55).

Theorem 11. For $n \geq 0$, the $n$th order q-Legendre polynomial is given by

$$
\widetilde{P}_{n}(q ; \omega)=\sum_{k=0}^{\lfloor n / 2\rfloor} C_{n, n-2 k} \omega^{n-2 k} q^{k(k+1)} \widetilde{P}_{0}\left(q ; \frac{\omega}{q^{k}}\right),
$$

where $C_{n, n-2 k}$ is the coefficient of $\omega^{n-2 k}$ in the nth degree Legendre polynomial $P_{n}(\omega)$, as given by (104) and (105).

Proof. Note that (109) is true in the $n=0$ case as it is a tautology, and it has been shown to hold for $1 \leq n \leq 4$ via (50)-(55). Assume that (109) has been established up through order $n$. Then, the recursion relation (34) expressed in terms of (109) gives that

$$
\begin{aligned}
& \widetilde{P}_{n+1}(q ; \omega)=\frac{2 n+1}{n+1} \omega \widetilde{P}_{n}(q ; \omega)-\frac{n}{n+1} q^{n+1} \widetilde{P}_{n-1}\left(q ; \frac{\omega}{q}\right) \\
&= \sum_{k=0}^{\lfloor n / 2\rfloor} \frac{2 n+1}{n+1} C_{n, n-2 k} \omega^{n+1-2 k} q^{k(k+1)} \widetilde{P}_{0}\left(q ; \frac{\omega}{q^{k}}\right) \\
&-\sum_{k=0}^{\lfloor(n-1) / 2\rfloor}\left\{\frac{n}{n+1} C_{n-1, n-1-2 k}\left(\frac{\omega}{q}\right)^{n-1-2 k}\right. \\
&= \sum_{k=0}^{\lfloor n / 2\rfloor} \frac{2 n+1}{n+1} C_{n, n-2 k} \omega^{n+1-2 k} q^{k(k+1)} \widetilde{P}_{0}\left(q ; \frac{\omega}{q^{k}}\right) \\
&-\sum_{k=0}^{\lfloor(n-1) / 2\rfloor}\left\{\frac{n}{n+1} C_{n-1, n-1-2 k} \omega^{n-1-2 k}\right. \\
&\left.\times q^{(k+2)(k+1)} \widetilde{P}_{0}\left(q ; \frac{\omega}{q^{k+1}}\right)\right\} C_{n, n-2 k} \omega^{n+1-2 k} q^{k(k+1)} \widetilde{P}_{0}\left(q ; \frac{\omega}{q^{k}}\right) \\
&\left.\sum^{k+1)} \widetilde{P}_{0}\left(q ; \frac{\omega}{q^{k+1}}\right)\right\} \\
&=
\end{aligned}
$$




$$
\begin{aligned}
& -\sum_{k=1}^{\lfloor(n-1) / 2\rfloor+1}\left\{\frac{n}{n+1} C_{n-1, n+1-2 k} \omega^{n+1-2 k}\right. \\
& \left.\times q^{k(k+1)} \widetilde{P}_{0}\left(q ; \frac{\omega}{q^{k}}\right)\right\} \\
& =\sum_{k=0}^{\lfloor(n+1) / 2\rfloor} C_{n+1, n+1-2 k} \omega^{n+1-2 k} q^{k(k+1)} \widetilde{P}_{0}\left(q ; \frac{\omega}{q^{k}}\right),
\end{aligned}
$$

where consolidating powers of $q$ gives (111), a reindexing on the subtracted summation in (111) gives (113), and the recursion relation obtained from setting (106) equal to (108) gives (114). Thus, (109) holds by induction.

Remark 12 . The utility of representing $\widetilde{P}_{n}(q ; \omega)$ by $(109)$ is that there are no nested integrals in (109), whereas the previous expressions (22) for higher order $\widetilde{P}_{n}(q ; \omega)$ involve nested integrals. Thus, we have gained computational efficiency.

Replacing each $\widetilde{P}_{0}\left(q ; \omega / q^{k}\right)$ in (109) by the corresponding integral expression in (21) yields the following.

Corollary 13. For $n \geq 0$, the $n$th order $q$-Legendre polynomial is given by

$$
\begin{aligned}
\widetilde{P}_{n}(q ; \omega)=\frac{-2\left(\mu_{q^{2}}\right)^{3}}{\pi f_{0, q}(0)} \sum_{k=0}^{\lfloor n / 2\rfloor}\left\{C_{n, n-2 k} \omega^{n-2 k} q^{k(k+1)}\right. \\
\left.\times \int_{-\infty}^{\omega / q^{k}} \frac{v}{\theta\left(q^{2} ; v^{2}\right)} d v\right\} .
\end{aligned}
$$

\section{Convergence Results for $\widetilde{P}_{n}(q ; \omega)$}

On closed sets not containing \pm 1 , one has uniform convergence of $\widetilde{P}_{n}(q ; \omega)$ to $P_{n}(\omega) \widetilde{\chi}_{[-1,1]}(\omega)$ as $q \rightarrow 1^{+}$. We will see that, via expression (109), one can obtain this result by relying on the fact that $\widetilde{P}_{0}(q ; \omega)$ converges uniformly to $P_{0}(\omega) \widetilde{\chi}_{[-1,1]}(\omega)$ away from \pm 1 . These results, coupled with an application of the reciprocal symmetry (71), will let us obtain pointwise convergence on all of $\mathbb{R}$.

9.1. Uniform Convergence Away from \pm 1 . First, we obtain a uniform convergence for $\widetilde{P}_{0}(q ; \omega)$ on subsets of the form $[-1+$ $\varepsilon, 1-\varepsilon]$.

Proposition 14. Given $0<\varepsilon<1$, one has that $\widetilde{P}_{0}(q ; \omega)$ converges uniformly to $P_{0}(\omega) \tilde{\chi}_{[-1,1]}(\omega)=1$ on the interval $[-1+\varepsilon, 1-\varepsilon]$ as $q \rightarrow 1^{+}$.

Proof. From (21), it follows that

$$
\frac{d}{d \omega} \widetilde{P}_{0}(q ; \omega)=\frac{-2\left(\mu_{q^{2}}\right)^{3}}{\pi f_{0, q}(0)} \frac{\omega}{\theta\left(q^{2} ; \omega^{2}\right)},
$$

from which we see that $\widetilde{P}_{0}(q ; \omega)$ is increasing on $(-\infty, 0)$ and decreasing on $(0, \infty)$ with a maximum at $\omega=0$ of

$$
\begin{aligned}
\widetilde{P}_{0}(q ; 0) & =\frac{-2\left(\mu_{q^{2}}\right)^{3}}{\pi f_{0, q}(0)} \int_{-\infty}^{0} \frac{\omega}{\theta\left(q^{2} ; \omega^{2}\right)} d \omega \\
& =\frac{2\left(\mu_{q^{2}}\right)^{3}}{\pi f_{0, q}(0)} \int_{0}^{\infty} \frac{\omega}{\theta\left(q^{2} ; \omega^{2}\right)} d \omega .
\end{aligned}
$$

From (21), along with the oddness of $\omega / \theta\left(q^{2} ; \omega^{2}\right)$, we have that $\widetilde{P}_{0}(q ; \omega)$ is even and $\lim _{\omega \rightarrow \pm \infty} \widetilde{P}_{0}(q ; \omega)=0$. Thus,

$$
0<\widetilde{P}_{0}(q ; \omega) \leq \widetilde{P}_{0}(q ; 0)
$$

for all $\omega \in \mathbb{R}$. Furthermore, on the interval $[-1+\varepsilon, 0]$, the function $\left|\widetilde{P}_{0}(q ; \omega)-P_{0}(\omega) \widetilde{\chi}_{[-1,1]}(\omega)\right|=\left|\widetilde{P}_{0}(q ; \omega)-1\right|$ assumes its maximum value at an endpoint $\omega=-1+\varepsilon$ or $\omega=0$.

From Proposition 19, we have that, given $\varepsilon_{1}>0$, there is a $Q_{2}>1$ such that for all $1<q<Q_{2}$

$$
\left|\widetilde{P}_{0}(q ;-1+\varepsilon)-1\right|<\varepsilon_{1} .
$$

From Proposition 20, we have that, given $\varepsilon_{1}>0$, there is a $Q_{1}>1$ with

$$
\left|\widetilde{P}_{0}(q ; 0)-1\right|=\widetilde{P}_{0}(q ; 0)-1<\varepsilon_{1}
$$

for all $1<q<Q_{1}$. From the increasing property of $\widetilde{P}_{0}(q ; \omega)$ on $[-1+\varepsilon, 0]$, we have

$$
\begin{aligned}
&\left|\widetilde{P}_{0}(q ; \omega)-P_{0}(\omega) \widetilde{\chi}_{[-1,1]}(\omega)\right|=\left|\widetilde{P}_{0}(q ; \omega)-1\right| \\
& \leq \max \left\{\left|\widetilde{P}_{0}(q ;-1+\varepsilon)-1\right|,\right. \\
&\left.\left|\widetilde{P}_{0}(q ; 0)-1\right|\right\}<\varepsilon_{1},
\end{aligned}
$$

for all $q$ with $1<q<\min \left\{Q_{1}, Q_{2}\right\}$. By evenness, (121) also gives uniform convergence on the interval $[-1+\varepsilon, 1-\varepsilon]$, and the proposition is proven.

Next, we obtain uniform convergence on closed sets not containing \pm 1 .

Theorem 15. Given $\varepsilon$ with $1>\varepsilon>0$, let $S_{\varepsilon} \equiv(-\infty,-1-\varepsilon] \cup$ $[-1+\varepsilon, 1-\varepsilon] \cup[1+\varepsilon, \infty)$. Then $\widetilde{P}_{n}(q ; \omega)$ converges uniformly to $P_{n}(\omega) \tilde{\chi}_{[-1,1]}(\omega)$ on $S_{\varepsilon}$ as $q \rightarrow 1^{+}$.

Proof. The proof breaks into two parts: (1) uniform convergence on the middle, that is on $[-1+\varepsilon, 1-\varepsilon]$, and (2) uniform convergence on the tails, that is on $(-\infty,-1-\varepsilon] \cup[1+\varepsilon, \infty)$.

We first handle the middle case. From Proposition 14, $\widetilde{P}_{0}(q ; \omega)$ converges uniformly to $1=P_{0}(\omega) \tilde{\chi}_{[-1,1]}(\omega)$ on the 
interval $[-1+\varepsilon, 1-\varepsilon]$ as $q \rightarrow 1^{+}$. From (104) and (105), one has that on $[-1+\varepsilon, 1-\varepsilon]$

$$
\begin{aligned}
\left|P_{n}(\omega)\right| & =\left|\sum_{k=0}^{\lfloor n / 2\rfloor} C_{n, n-2 k} \omega^{n-2 k}\right| \\
& \leq \sum_{k=0}^{\lfloor n / 2\rfloor}\left|C_{n, n-2 k}\right||\omega|^{n-2 k} \\
& <\sum_{k=0}^{\lfloor n / 2\rfloor}\left|C_{n, n-2 k}\right|(1)^{n-2 k} \equiv A_{n} .
\end{aligned}
$$

Thus, given $\varepsilon_{1}$ with $1>\varepsilon_{1}>0$, there is $q_{1}>1$ such that

$$
\left|\widetilde{P}_{0}(q ; \omega)-1\right|<\frac{\varepsilon_{1}}{2 A_{n}}
$$

for all $q$ with $1<q<q_{1}$ and for all $\omega$ with $|\omega| \leq 1-\varepsilon$. Then, for each $\omega \in[-1+\varepsilon, 1-\varepsilon]$ and $k \geq 0$, one has $\omega / q^{k} \in[-1+\varepsilon, 1-\varepsilon]$, given that

$$
\left|\widetilde{P}_{0}\left(q ; \frac{\omega}{q^{k}}\right)-1\right|<\frac{\varepsilon_{1}}{2 A_{n}}
$$

for all $1<q<q_{1}$. Thus, for $\omega \in[-1+\varepsilon, 1-\varepsilon]$ and $1<q<q_{1}$, by relying on (109) and (104), we have

$$
\begin{aligned}
& \left|\widetilde{P}_{n}(q ; \omega)-P_{n}(\omega) \tilde{\chi}_{[-1,1]}(\omega)\right| \\
& =\mid \sum_{k=0}^{\lfloor n / 2\rfloor} C_{n, n-2 k} \omega^{n-2 k} q^{k(k+1)} \widetilde{P}_{0}\left(q ; \frac{\omega}{q^{k}}\right) \\
& -\sum_{k=0}^{\lfloor n / 2\rfloor} C_{n, n-2 k} \omega^{n-2 k} \tilde{\chi}_{[-1,1]}(\omega) \\
& \leq \mid \sum_{k=0}^{\lfloor n / 2\rfloor} C_{n, n-2 k} \omega^{n-2 k} q^{k(k+1)} \widetilde{P}_{0}\left(q ; \frac{\omega}{q^{k}}\right) \\
& -\sum_{k=0}^{\lfloor n / 2\rfloor} C_{n, n-2 k} \omega^{n-2 k} \widetilde{P}_{0}\left(q ; \frac{\omega}{q^{k}}\right) \\
& +\mid \sum_{k=0}^{\lfloor n / 2\rfloor} C_{n, n-2 k} \omega^{n-2 k} \widetilde{P}_{0}\left(q ; \frac{\omega}{q^{k}}\right) \\
& -\sum_{k=0}^{\lfloor n / 2\rfloor} C_{n, n-2 k} \omega^{n-2 k} \tilde{\chi}_{[-1,1]}(\omega) \mid \\
& \leq \sum_{k=0}^{\lfloor n / 2\rfloor}\left|C_{n, n-2 k}\right||\omega|^{n-2 k}\left(q^{k(k+1)}-1\right)\left|\widetilde{P}_{0}\left(q ; \frac{\omega}{q^{k}}\right)\right| \\
& +\sum_{k=0}^{\lfloor n / 2\rfloor}\left|C_{n, n-2 k}\right||\omega|^{n-2 k}\left|\widetilde{P}_{0}\left(q ; \frac{\omega}{q^{k}}\right)-1\right| \\
& <\sum_{k=0}^{\lfloor n / 2\rfloor}\left|C_{n, n-2 k}\right|(1)^{n-2 k}\left(q^{(\lfloor n / 2\rfloor)(\lfloor n / 2\rfloor+1)}-1\right)\left(1+\frac{\varepsilon_{1}}{2 A_{n}}\right) \\
& +\sum_{k=0}^{\lfloor n / 2\rfloor}\left|C_{n, n-2 k}\right|(1)^{n-2 k} \frac{\varepsilon_{1}}{2 A_{n}}
\end{aligned}
$$

$$
\begin{aligned}
& =A_{n}\left(q^{(\lfloor n / 2\rfloor)(\lfloor n / 2\rfloor+1)}-1\right)\left(1+\frac{\varepsilon_{1}}{2 A_{n}}\right)+A_{n} \frac{\varepsilon_{1}}{2 A_{n}} \\
& =\left(q^{(\lfloor n / 2\rfloor)(\lfloor n / 2\rfloor+1)}-1\right)\left(A_{n}+\frac{\varepsilon_{1}}{2}\right)+\frac{\varepsilon_{1}}{2} .
\end{aligned}
$$

When

$$
\left(q^{(\lfloor n / 2\rfloor)(\lfloor n / 2\rfloor+1)}-1\right)\left(A_{n}+\frac{\varepsilon_{1}}{2}\right)<\frac{\varepsilon_{1}}{2}
$$

we see that (126) is less than $\varepsilon_{1}$. However, (127) holds when

$$
\left(q^{(\lfloor n / 2\rfloor)(\lfloor n / 2\rfloor+1)}-1\right)<\frac{\varepsilon_{1}}{2\left(A_{n}+\varepsilon_{1} / 2\right)},
$$

which is true for all $q<q_{2}$ with

$$
q_{2}=\left(1+\frac{\varepsilon_{1}}{2 A_{n}+1}\right)^{1 /[(\lfloor n / 2\rfloor)(\lfloor n / 2\rfloor+1)]} .
$$

Thus, given $1>\varepsilon_{1}>0$, for all $q$ with $1<q<\min \left\{q_{1}, q_{2}\right\}$ from (126) and (127), one has

$$
\left|\widetilde{P}_{n}(q ; \omega)-P_{n}(\omega) \tilde{\chi}_{[-1,1]}(\omega)\right|<\varepsilon_{1}
$$

for all $\omega \in[-1+\varepsilon, 1-\varepsilon]$. Thus, uniform convergence is obtained on $[-1+\varepsilon, 1-\varepsilon]$, and part 1 of the proof is shown.

We proceed to part 2 of the proof, uniform convergence in the tails $|\omega| \geq 1+\varepsilon$. Let $\varepsilon_{1}>0$ be given. Then, for $\omega \in$ $(-\infty,-1-\varepsilon] \cup[1+\varepsilon, \infty)$, one has

$$
\begin{aligned}
\mid \widetilde{P}_{n} & (q ; \omega)-P_{n}(\omega) \widetilde{\chi}_{[-1,1]}(\omega) \mid \\
= & \left|\sum_{k=0}^{\lfloor n / 2\rfloor} C_{n, n-2 k} q^{k(k+1)} \omega^{n-2 k} \widetilde{P}_{0}\left(q ; \frac{\omega}{q^{k}}\right)-0\right| \\
\leq & \sum_{k=0}^{\lfloor n / 2\rfloor}\left|C_{n, n-2 k}\right| q^{k(k+1)}\left|\omega^{n-2 k} \widetilde{P}_{0}\left(q ; \frac{\omega}{q^{k}}\right)\right| \\
\leq & {\left[\frac{2\left(\mu_{q^{2}}\right)^{3} \ln (q)}{\pi f_{0, q}(0)}\right]\{\sqrt{\pi}-\sqrt{\ln (q)}\}^{-1} \sqrt{\frac{\pi}{2}} } \\
& \cdot \sum_{k=0}^{\lfloor n / 2\rfloor}\left\{\left|C_{n, n-2 k}\right| q^{k(k+1)} q^{\left[(n-2 k)^{2}+2(n-2 k)(2 k+1)\right] / 4}\right. \\
& \times e^{-[\ln (|\omega|)-(n+1) \ln (q) / 2]^{2} / \ln (q)} \\
& \cdot q^{n(n+2) / 4}\left(\sum_{k=0}^{-[\ln (|\omega|)-(n-2 k+2 k+1) \ln (q) / 2]^{2} / \ln (q)}\right\} \\
& {\left[\frac{2\left(\mu_{q^{2}}\right)^{3} \ln (q)}{\pi f_{0, q}(0)}\right]\{\sqrt{\pi}-\sqrt{\ln (q)}\}^{-1} \sqrt{\frac{\pi}{2}} } \\
& {[0,2 k) } \\
&
\end{aligned}
$$




$$
\begin{aligned}
\leq & {\left[\frac{2\left(\mu_{q^{2}}\right)^{3} \ln (q)}{\pi f_{0, q}(0)}\right]\{\sqrt{\pi}-\sqrt{\ln (q)}\}^{-1} \sqrt{\frac{\pi}{2}} } \\
& \cdot q^{n(n+2) / 4}\left(\sum_{k=0}^{\lfloor n / 2\rfloor}\left|C_{n, n-2 k}\right|\right) \\
& \times e^{-\left[\ln \left(\left|\omega_{0}\right|\right)-(n+1) \ln (q) / 2\right]^{2} / \ln (q)},
\end{aligned}
$$

where (132) follows from (143) of Proposition 17, with $p=$ $n-2 k$, and holds for $1<q<q_{3}$ and $|\omega| \geq\left|\omega_{0}\right|$ with

$$
1<q_{3}^{(n-2 k+2 k+1) / 2}=q_{3}^{(n+1) / 2}<\left|\omega_{0}\right|<1+\varepsilon
$$

as obtained in (154).

From (17), one has

$$
\begin{aligned}
& \lim _{q \rightarrow 1^{+}}\left[\frac{2\left(\mu_{q^{2}}\right)^{3} \ln (q)}{\pi f_{0, q}(0)}\right]=1, \\
& \lim _{q \rightarrow 1^{+}}\{\sqrt{\pi}-\sqrt{\ln (q)}\}^{-1} \sqrt{\frac{\pi}{2}} q^{n(n+2) / 4}=\frac{1}{\sqrt{2}},
\end{aligned}
$$

from which it follows via (136) that there is a $q_{4}$ with $1<q_{4}<$ $q_{3}$ such that for all $1<q<q_{4}$

$$
\begin{aligned}
\left|\widetilde{P}_{n}(q ; \omega)-P_{n}(\omega) \widetilde{\chi}_{[-1,1]}(\omega)\right| \leq & 1\left(\sum_{k=0}^{\lfloor n / 2\rfloor}\left|C_{n, n-2 k}\right|\right) \\
& \times e^{-\left[\ln \left(\left|\omega_{0}\right|\right)-(n+1) \ln (q) / 2\right]^{2} / \ln (q)}
\end{aligned}
$$

for all $|\omega| \geq\left|\omega_{0}\right|$. Since

$$
\lim _{q \rightarrow 1^{+}} e^{-\left[\ln \left(\left|\omega_{0}\right|\right)-(n+1) \ln (q) / 2\right]^{2} / \ln (q)}=0
$$

there is a $q_{5}$ with $1<q_{5}<q_{4}$ such that

$$
\left|\widetilde{P}_{n}(q ; \omega)-P_{n}(\omega) \tilde{\chi}_{[-1,1]}(\omega)\right|<\varepsilon_{1}
$$

for all $1<q<q_{5}$ and all $|\omega| \geq\left|\omega_{0}\right|$. Thus, since $1+\varepsilon>$ $\left|\omega_{0}\right|$, from (137), we have uniform convergence in the tails, finishing the proof of part 2 and concluding the proof of the theorem.

Remark 16. The decay expressed in (134) gives a stronger result than uniform convergence, as is shown in Section 10.

The following proposition was utilized in showing part 2 of Theorem 15.
Proposition 17. Given $p, k \in \mathbb{N}_{0}$ and given $\varepsilon>0$, then for each $\omega_{0}$ with $1<\omega_{0}<1+\varepsilon$ there exists $q_{3}>1$ such that

$$
\begin{aligned}
& \left|\omega^{p} \widetilde{P}_{0}\left(q ; \frac{\omega}{q^{k}}\right)\right| \\
& \leq\left[\frac{2\left(\mu_{q^{2}}\right)^{3} \ln (q)}{\pi f_{0, q}(0)}\right]\{\sqrt{\pi}-\sqrt{\ln (q)}\}^{-1} \\
& \quad \times \sqrt{\frac{\pi}{2}} q^{\left[p^{2}+2 p(2 k+1)\right] / 4} e^{-[\ln (|\omega|)-(p+2 k+1) \ln (q) / 2]^{2} / \ln (q)} \\
& \leq\left[\frac{2\left(\mu_{q^{2}}\right)^{3} \ln (q)}{\pi f_{0, q}(0)}\right]\{\sqrt{\pi}-\sqrt{\ln (q)}\}^{-1} \\
& \quad \times \sqrt{\frac{\pi}{2}} q^{\left[p^{2}+2 p(2 k+1)\right] / 4} e^{-\left[\ln \left(\left|\omega_{0}\right|\right)-(p+2 k+1) \ln (q) / 2\right]^{2} / \ln (q)}
\end{aligned}
$$

for all $1<q<q_{3}$ and all $|\omega| \geq \omega_{0}$.

Proof. From (21), one has

$$
\begin{aligned}
& \left|\omega^{p} \widetilde{P}_{0}\left(q ; \frac{\omega}{q^{k}}\right)\right| \\
& =\left|\omega^{p} \frac{2\left(\mu_{q^{2}}\right)^{3}}{\pi f_{0, q}(0)} \int_{-\infty}^{\omega / q^{k}} \frac{v}{\theta\left(q^{2} ; v^{2}\right)} d v\right| \\
& =|\omega|^{p} \frac{2\left(\mu_{q^{2}}\right)^{3}}{\pi f_{0, q}(0)}\left|\int_{-\infty}^{-|\omega| / q^{k}} \frac{v}{\theta\left(q^{2} ; v^{2}\right)} d v\right| \\
& =|\omega|^{p} \frac{2\left(\mu_{q^{2}}\right)^{3}}{\pi f_{0, q}(0)} \int_{|\omega| / q^{k}}^{\infty} \frac{v}{\theta\left(q^{2} ; v^{2}\right)} d v \\
& \leq|\omega|^{p} \frac{2\left(\mu_{q^{2}}\right)^{3}}{\pi f_{0, q}(0)} q^{-1 / 4}\left\{\sqrt{\frac{\pi}{\ln (q)}}-1\right\}^{-1} \\
& \times \int_{|\omega| / q^{k}}^{\infty} e^{-[\ln (v)]^{2} / \ln (q)} d v \\
& \leq|\omega|^{p} \frac{2\left(\mu_{q^{2}}\right)^{3}}{\pi f_{0, q}(0)} q^{-1 / 4}\left\{\sqrt{\frac{\pi}{\ln (q)}}-1\right\}^{-1} \\
& \times \sqrt{\frac{\pi}{2}} \sqrt{\ln (q)} e^{[\ln (q)] / 4} e^{-\left[\ln \left(|\omega| / q^{k}\right)-\ln (q) / 2\right]^{2} / \ln (q)} \\
& =e^{p \ln (|\omega|)}\left[\frac{2\left(\mu_{q^{2}}\right)^{3} \ln (q)}{\pi f_{0, q}(0)}\right]\{\sqrt{\ln (q)}\}^{-1} \\
& \times\left\{\sqrt{\frac{\pi}{\ln (q)}}-1\right\}^{-1} \sqrt{\frac{\pi}{2}} e^{-[\ln (|\omega|)-(k+1 / 2) \ln (q)]^{2} / \ln (q)}
\end{aligned}
$$




$$
\begin{aligned}
= & {\left[\frac{2\left(\mu_{q^{2}}\right)^{3} \ln (q)}{\pi f_{0, q}(0)}\right]\{\sqrt{\pi}-\sqrt{\ln (q)}\}^{-1} } \\
& \times \sqrt{\frac{\pi}{2}} e^{-\left[\ln ^{2}(|\omega|)-(p+2 k+1) \ln (|\omega|) \ln (q)+(k+1 / 2)^{2} \ln ^{2}(q)\right] / \ln (q)} \\
= & {\left[\frac{2\left(\mu_{q^{2}}\right)^{3} \ln (q)}{\pi f_{0, q}(0)}\right]\{\sqrt{\pi}-\sqrt{\ln (q)}\}^{-1} } \\
& \times \sqrt{\frac{\pi}{2}} q^{\left[p^{2}+2 p(2 k+1)\right] / 4} e^{-[\ln (|\omega|)-(p+2 k+1) \ln (q) / 2]^{2} / \ln (q)}
\end{aligned}
$$

Here, (146) follows from oddness of the integrand $v / \theta\left(q^{2} ; v^{2}\right)$. Then, (147) follows from the bound (27). Also, (148) follows from the bound

$$
\int_{C}^{\infty} e^{-A[\ln (x)+B]^{2}} d x \leq \frac{\sqrt{\pi}}{2} \frac{e^{1 /(4 A)-B}}{\sqrt{A}} e^{-A[\ln (C)+B-1 /(2 A)]^{2}}
$$

for $C>e^{-B+1 /(2 A)}$, from $[4,14]$, with $A=1 / \ln (q), B=0$, and $C=|\omega| / q^{k}$. Finally, (150) follows by a completion of squares. Note that the requirement that $C>e^{-B+1 /(2 A)}$ in (151) becomes $|\omega| / q^{k}>e^{\ln (q) / 2}$ or $|\omega|>q^{k+1 / 2}$ in (148). However, in order for (150) to be decreasing in $|\omega|$, we require the slightly stronger condition that

$$
\ln (|\omega|)-\frac{(p+2 k+1) \ln (q)}{2}>0,
$$

or equivalently

$$
|\omega|>q^{(p+2 k+1) / 2} .
$$

Now, given $\varepsilon>0$, and $p, k \in \mathbb{N}_{0}$ as in the hypotheses of the proposition, first choose $\omega_{0}$ with $1<\omega_{0}<1+\varepsilon$ and then choose $q_{3}$ with $1<q_{3}<\left|\omega_{0}\right|^{2 /[p+2 k+1]}$ so that

$$
1<q_{3}^{(p+2 k+1) / 2}<\left|\omega_{0}\right|<1+\varepsilon
$$

Then, for $1<q<q_{3}$ and $|\omega| \geq\left|\omega_{0}\right|$, one has that (153) holds, from which (150) is decreasing in $|\omega|$. This then gives (143) and (144) and completes the proof of the proposition.

9.2. Pointwise Convergence on $\mathbb{R}$. It is now possible to give a pointwise convergence result.

Theorem 18. $\widetilde{P}_{n}(q ; \omega)$ converges pointwise to $P_{n}(\omega) \widetilde{\chi}_{[-1,1]}(\omega)$ as $q \rightarrow 1^{+}$.

Proof. By virtue of Theorem 15, one automatically has pointwise convergence for $\omega \neq \pm 1$. Thus, we concentrate on $\omega= \pm 1$ to obtain the result. Observe first that for any $p \in \mathbb{R}$ one has

$$
\lim _{q \rightarrow 1^{+}}\left[\widetilde{P}_{0}\left(q ; q^{p}\right)-\widetilde{P}_{0}(q ; 1)\right]=0
$$

This follows from the estimate

$$
\begin{aligned}
& \left|\widetilde{P}_{0}\left(q ; q^{p}\right)-\widetilde{P}_{0}(q ; 1)\right| \\
& =\mid \frac{-2}{\pi} \frac{\left(\mu_{q^{2}}\right)^{3}}{f_{0, q}(0)} \int_{-\infty}^{q^{p}} \frac{\omega}{\theta\left(q^{2} ; \omega^{2}\right)} d \omega \\
& -\frac{-2}{\pi} \frac{\left(\mu_{q^{2}}\right)^{3}}{f_{0, q}(0)} \int_{-\infty}^{1} \frac{\omega}{\theta\left(q^{2} ; \omega^{2}\right)} d \omega \\
& =\frac{2}{\pi} \frac{\left(\mu_{q^{2}}\right)^{3}}{f_{0, q}(0)}\left|\int_{1}^{q^{p}} \frac{\omega}{\theta\left(q^{2} ; \omega^{2}\right)} d \omega\right| \\
& \leq \frac{2}{\pi} \frac{\ln (q)\left(\mu_{q^{2}}\right)^{3}}{f_{0, q}(0)} \frac{q^{-1 / 4}}{\{\sqrt{\pi}-\sqrt{\ln (q)}\}} \\
& \times\left[\frac{1}{\sqrt{\ln (q)}}\left|\int_{1}^{q^{p}} \frac{1}{e^{(\ln (|\omega|))^{2} / \ln (q)}} d \omega\right|\right] \\
& \leq \frac{2}{\pi} \frac{\ln (q)\left(\mu_{q^{2}}\right)^{3}}{f_{0, q}(0)} \frac{q^{-1 / 4}}{\{\sqrt{\pi}-\sqrt{\ln (q)}\}} \\
& \times\left[\frac{1}{\sqrt{\ln (q)}}\left|q^{p}-1\right|\right],
\end{aligned}
$$

where (27) was used to obtain (157) and where the integrand in (157) being at most 1 was used to obtain (158). Now, by L'Hopital's rule, one has, for each $p \in \mathbb{R}$, that

$$
\begin{aligned}
\lim _{q \rightarrow 1^{+}} \frac{q^{p}-1}{\sqrt{\ln (q)}} & =\lim _{q \rightarrow 1^{+}} \frac{p q^{p-1}}{(1 / 2)(\ln (q))^{-1 / 2}(1 / q)} \\
& =\lim _{q \rightarrow 1^{+}} 2 p q^{p} \sqrt{\ln (q)}=0 .
\end{aligned}
$$

By the $q$-Wallis limit (17), one also has

$$
\lim _{q \rightarrow 1^{+}} \frac{2}{\pi} \frac{\ln (q)\left(\mu_{q^{2}}\right)^{3}}{f_{0, q}(0)} \frac{q^{-1 / 4}}{\{\sqrt{\pi}-\sqrt{\ln (q)}\}}=\frac{1}{\sqrt{\pi}} .
$$

Applying (159) and (160) to (158) gives (155).

Next, observe from the reciprocal identity (71) with $\omega$ set equal to 1 , that

$$
\widetilde{P}_{0}(q ; 0)-\widetilde{P}_{0}(q ; \sqrt{q})=\widetilde{P}_{0}(q ; \sqrt{q})
$$

from which one obtains

$$
\frac{1}{2} \widetilde{P}_{0}(q ; 0)=\widetilde{P}_{0}(q ; \sqrt{q}) .
$$


Proposition 20 gives that $\lim _{q \rightarrow 1^{+}} \widetilde{P}_{0}(q ; 0)=1$. Applying this to (162) gives

$$
\lim _{q \rightarrow 1^{+}} \widetilde{P}_{0}(q ; \sqrt{q})=\frac{1}{2} \lim _{q \rightarrow 1^{+}} \widetilde{P}_{0}(q ; 0)=\frac{1}{2}
$$

Setting $p=1 / 2$ in (155) gives

$$
\lim _{q \rightarrow 1^{+}} \widetilde{P}_{0}(q ; 1)=\lim _{q \rightarrow 1^{+}} \widetilde{P}_{0}(q ; \sqrt{q})=\frac{1}{2} .
$$

Letting $p$ be arbitrary in (155) gives

$$
\lim _{q \rightarrow 1^{+}} \widetilde{P}_{0}\left(q ; q^{p}\right)=\lim _{q \rightarrow 1^{+}} \widetilde{P}_{0}(q ; 1)=\frac{1}{2}
$$

for all $p \in \mathbb{R}$.

Next, from (109), we have

$$
\widetilde{P}_{n}(q ; 1)=\sum_{k=0}^{\lfloor n / 2\rfloor} C_{n, n-2 k} 1^{n-2 k} q^{k(k+1)} \widetilde{P}_{0}\left(q ; \frac{1}{q^{k}}\right) .
$$

From (165), one has that $\lim _{q \rightarrow 1^{+}} \widetilde{P}_{0}\left(q ; 1 / q^{k}\right)=1 / 2$. Taking limits of (166) with this in mind yields

$$
\begin{aligned}
\lim _{q \rightarrow 1^{+}} \widetilde{P}_{n}(q ; 1) & =\sum_{k=0}^{\lfloor n / 2\rfloor} C_{n, n-2 k} 1^{n-2 k} \lim _{q \rightarrow 1^{+}}\left[q^{k(k+1)} \widetilde{P}_{0}\left(q ; \frac{1}{q^{k}}\right)\right] \\
& =\sum_{k=0}^{\lfloor n / 2\rfloor} C_{n, n-2 k} 1^{n-2 k} \frac{1}{2} \\
& =P_{n}(1) \frac{1}{2}=P_{n}(1) \widetilde{\chi}_{[-1,1]}(1)=\frac{1}{2}
\end{aligned}
$$

where (168) follows from (165) and (169) follows from (104). By evenness/oddness, respectively, one has

$$
\begin{aligned}
\lim _{q \rightarrow 1^{+}} \widetilde{P}_{n}(q ;-1) & =\lim _{q \rightarrow 1^{+}}\left[(-1)^{n} \widetilde{P}_{n}(q ; 1)\right] \\
& =(-1)^{n} P_{n}(1) \widetilde{\chi}_{[-1,1]}(1) \\
& =P_{n}(-1) \widetilde{\chi}_{[-1,1]}(-1)=(-1)^{n} \frac{1}{2} .
\end{aligned}
$$

Equations (169) and (170) give pointwise convergence at \pm 1 and the theorem is proven.

9.3. Estimates Giving Proposition 14. The propositions in this subsection provide the estimates on which Proposition 14 is based.

Proposition 19. For $0<\varepsilon<1$, given $\varepsilon_{1}>0$, there is a $Q_{2}>1$ such that

$$
\left|\widetilde{P}_{0}(q ;-1+\varepsilon)-1\right|<\varepsilon_{1}
$$

for all $1<q<Q_{2}$.
Proof.

$$
\begin{aligned}
\left|\widetilde{P}_{0}(q ;-1+\varepsilon)-1\right| \leq & \left|\widetilde{P}_{0}(q ;-1+\varepsilon)-\widetilde{P}_{0}(q ; 0)\right| \\
& +\left|\widetilde{P}_{0}(q ; 0)-1\right| .
\end{aligned}
$$

By virtue of Proposition 20 below, which bounds $\left|\widetilde{P}_{0}(q ; 0)-1\right|$, we only need bound the first term, $\left|\widetilde{P}_{0}(q ;-1+\varepsilon)-\widetilde{P}_{0}(q ; 0)\right|$, in the right-hand side of inequality (172).

Evenness of $\widetilde{P}_{0}(q ; \omega)$ and the setting of $\omega=-1+\varepsilon$ in the reciprocal identity (70) together give

$$
\begin{aligned}
0 & <\widetilde{P}_{0}(q ; 0)-\widetilde{P}_{0}(q ;-1+\varepsilon) \\
& =\widetilde{P}_{0}\left(q ; \frac{q}{(-1+\varepsilon)}\right)=\widetilde{P}_{0}\left(q ; \frac{q}{(1-\varepsilon)}\right),
\end{aligned}
$$

for all $q>1$. Now, since $1+\varepsilon<1 /(1-\varepsilon)<q /(1-\varepsilon)$ and $\widetilde{P}_{0}(q ; \omega)$ is decreasing for $\omega>0$, we have that $\widetilde{P}_{0}(q ; 1+\varepsilon)>$ $\widetilde{P}_{0}(q ; 1 /(1-\varepsilon))>\widetilde{P}_{0}(q ; q /(1-\varepsilon))$, and this applied to $(173)$ yields

$$
\begin{aligned}
0 & <\widetilde{P}_{0}(q ; 0)-\widetilde{P}_{0}(q ;-1+\varepsilon) \\
& =\widetilde{P}_{0}\left(q ; \frac{q}{(1-\varepsilon)}\right)<\widetilde{P}_{0}(q ; 1+\varepsilon) .
\end{aligned}
$$

Next, pick $\omega_{0}$ with $1<\omega_{0}<1+\varepsilon$, and apply Proposition 17 with $p=k=0$ and $\omega=1+\varepsilon$ to obtain

$$
\begin{aligned}
\widetilde{P}_{0}(q ; 1+\varepsilon) \leq & {\left[\frac{2\left(\mu_{q^{2}}\right)^{3} \ln (q)}{\pi f_{0, q}(0)}\right]\{\sqrt{\pi}-\sqrt{\ln (q)}\}^{-1} } \\
& \times \sqrt{\frac{\pi}{2}} e^{-\left[\ln \left(\left|\omega_{0}\right|\right)-\ln (q) / 2\right]^{2} / \ln (q)}
\end{aligned}
$$

By (17), one has

$$
\lim _{q \rightarrow 1+}\left[\frac{2\left(\mu_{q^{2}}\right)^{3} \ln (q)}{\pi f_{0, q}(0)}\right]\{\sqrt{\pi}-\sqrt{\ln (q)}\}^{-1} \sqrt{\frac{\pi}{2}}=\frac{1}{\sqrt{2}}
$$

and one sees directly that

$$
\lim _{q \rightarrow 1+} e^{-\left[\ln \left(\left|\omega_{0}\right|\right)-\ln (q) / 2\right]^{2} / \ln (q)}=0
$$

Thus, the right-hand side of (175) approaches 0 as $q \rightarrow 1^{+}$. So, from (175) and (174), given $\varepsilon_{1}>0$, there is a $Q_{2}>1$ such that, for all $1<q<Q_{2}$,

$$
0<\widetilde{P}_{0}(q ; 0)-\widetilde{P}_{0}(q ;-1+\varepsilon)<\widetilde{P}_{0}(q ; 1+\varepsilon)<\frac{\varepsilon_{1}}{2} .
$$

This gives the proposition. 
Next, by (29), we have

$$
\begin{aligned}
{ }_{q} \operatorname{Cos}(t) & =\mathscr{F}^{-1}\left(\mathscr{F}\left({ }_{q} \operatorname{Cos}(t)\right)\right) \\
& =\frac{1}{\sqrt{2 \pi}} \int_{-\infty}^{\infty} e^{i \omega t} \sqrt{\frac{2}{\pi}} \frac{\left(\mu_{q^{2}}\right)^{3}}{f_{0, q}(0)} \frac{1}{\theta\left(q^{2} ; \omega^{2}\right)} d \omega \\
& =\frac{1}{\pi} \frac{\left(\mu_{q^{2}}\right)^{3}}{f_{0, q}(0)} \int_{-\infty}^{\infty} e^{i \omega t} \frac{1}{\theta\left(q^{2} ; \omega^{2}\right)} d \omega,
\end{aligned}
$$

from which one obtains that

$$
\begin{aligned}
1 & ={ }_{q} \operatorname{Cos}(0)=\frac{1}{\pi} \frac{\left(\mu_{q^{2}}\right)^{3}}{f_{0, q}(0)} \int_{-\infty}^{\infty} \frac{1}{\theta\left(q^{2} ; \omega^{2}\right)} d \omega \\
& =\frac{2}{\pi} \frac{\left(\mu_{q^{2}}\right)^{3}}{f_{0, q}(0)} \int_{0}^{\infty} \frac{1}{\theta\left(q^{2} ; \omega^{2}\right)} d \omega .
\end{aligned}
$$

Proposition 20. For each $\varepsilon>0$, there exists a $Q>1$ such that for all $1<q<Q$

$$
0<\widetilde{P}_{0}(q ; 0)-1<\varepsilon .
$$

Proof. Subtracting (180) from (117), one has

$$
\begin{aligned}
\widetilde{P}_{0}(q ; 0)-1= & \frac{2\left(\mu_{q^{2}}\right)^{3}}{\pi f_{0, q}(0)} \int_{0}^{\infty} \frac{\omega-1}{\theta\left(q^{2} ; \omega^{2}\right)} d \omega \\
= & \frac{2\left(\mu_{q^{2}}\right)^{3}}{\pi f_{0, q}(0)} \\
& \times\left[\int_{0}^{1} \frac{\omega-1}{\theta\left(q^{2} ; \omega^{2}\right)} d \omega+\int_{1}^{\infty} \frac{\omega-1}{\theta\left(q^{2} ; \omega^{2}\right)} d \omega\right] .
\end{aligned}
$$

The change of variables $\omega=1 / u$ is made on the first integral in (182), and the algebraic identity $u^{2} \theta\left(q^{2} ; u^{-2}\right)=\theta\left(q^{2} ; u^{2}\right)$ is used to obtain

$$
\begin{aligned}
\int_{0}^{1} \frac{\omega-1}{\theta\left(q^{2} ; \omega^{2}\right)} d \omega & =\int_{\infty}^{1} \frac{u^{-1}-1}{\theta\left(q^{2} ; u^{-2}\right)} \frac{(-d u)}{u^{2}} \\
& =\int_{1}^{\infty} \frac{u^{-1}-1}{\theta\left(q^{2} ; u^{2}\right)} d u .
\end{aligned}
$$

Now (183) is used to reexpress (182) as

$$
\begin{aligned}
\widetilde{P}_{0}(q ; 0)-1 \\
=\frac{2\left(\mu_{q^{2}}\right)^{3}}{\pi f_{0, q}(0)}\left[\int_{1}^{\infty} \frac{\omega^{-1}-1}{\theta\left(q^{2} ; \omega^{2}\right)} d \omega+\int_{1}^{\infty} \frac{\omega-1}{\theta\left(q^{2} ; \omega^{2}\right)} d \omega\right] \\
=\frac{2\left(\mu_{q^{2}}\right)^{3}}{\pi f_{0, q}(0)} \int_{1}^{\infty} \frac{\omega^{-1}-2+\omega}{\theta\left(q^{2} ; \omega^{2}\right)} d \omega \\
=\frac{2\left(\mu_{q^{2}}\right)^{3}}{\pi f_{0, q}(0)} \int_{1}^{\infty} \frac{(\omega-1)^{2}}{\omega \theta\left(q^{2} ; \omega^{2}\right)} d \omega>0
\end{aligned}
$$

from which one sees that $\widetilde{P}_{0}(q ; 0)>1$ for all $q>1$. Deploying the bound (27) within the integral in (185) gives

$$
\begin{aligned}
0 & <\widetilde{P}_{0}(q ; 0)-1 \leq F(q)\left[\frac{1}{\sqrt{\ln (q)}} \int_{1}^{\infty} \frac{(\omega-1)^{2} \omega^{-2}}{e^{(\ln \omega)^{2} / \ln (q)}} d \omega\right] \\
& =F(q)\left[\frac{1}{\sqrt{\ln (q)}} \int_{1}^{\infty} \frac{\left(1-\omega^{-1}\right)^{2}}{e^{(\ln \omega)^{2} / \ln (q)}} d \omega\right],
\end{aligned}
$$

where

$$
F(q)=\frac{1}{q^{1 / 4}} \frac{2}{\pi}\left[\frac{\ln (q)\left(\mu_{q^{2}}\right)^{3}}{f_{0, q}(0)}\right] \frac{1}{\sqrt{\pi}-\sqrt{\ln (q)}} .
$$

It follows from the $q$-Wallis formula (17) that

$$
\lim _{q \rightarrow 1^{+}} F(q)=\frac{1}{\sqrt{\pi}} \text {. }
$$

We now show that $\widetilde{P}_{0}(q ; 0)-1$ can be made arbitrarily small for all $q>1$ sufficiently close to $1^{+}$. In the light of (188), this is accomplished by first showing that the corresponding statement holds for the bracketed expression in (186).

Let $\alpha>0$ be arbitrary, with $\alpha$ being specified later. The integral in $\omega$ over the interval $[1, \infty)$ in $(186)$ is now subdivided into two integrals, the first over $\left[1, e^{\alpha \sqrt{\ln (q)}}\right]$ and the second over $\left[e^{\alpha \sqrt{\ln (q)}}, \infty\right)$. First, on $\left[1, e^{\alpha \sqrt{\ln (q)}}\right]$, one computes

$$
\frac{1}{\sqrt{\ln (q)}} \int_{1}^{e^{\alpha \sqrt{\ln (q)}}} \frac{\left(1-\omega^{-1}\right)^{2}}{e^{(\ln \omega)^{2} / \ln (q)}} d \omega
$$

Now, the function $\left(1-\omega^{-1}\right)^{2}$ is increasing on $\left[1, e^{\alpha \sqrt{\ln (q)}}\right]$, and it assumes its maximum value of $\left(1-e^{-\alpha \sqrt{\ln (q)}}\right)^{2}$ at the right endpoint $e^{\alpha \sqrt{\ln (q)}}$. Thus, we bound the integral in (189) by the length of the interval times the bound $\left(1-e^{-\alpha \sqrt{\ln (q)}}\right)^{2}$ on the numerator. This gives

$$
\begin{aligned}
& \frac{1}{\sqrt{\ln (q)}} \int_{1}^{e^{\alpha \sqrt{\ln (q)}}} \frac{\left(1-\omega^{-1}\right)^{2}}{e^{(\ln \omega)^{2} / \ln (q)}} d \omega \\
& \leq \frac{1}{\sqrt{\ln (q)}} \int_{1}^{e^{\alpha \sqrt{\ln (q)}}}\left(1-e^{-\alpha \sqrt{\ln (q)}}\right)^{2} d \omega \\
& \leq \frac{1}{\sqrt{\ln (q)}}\left(e^{\alpha \sqrt{\ln (q)}}-1\right)\left(1-e^{-\alpha \sqrt{\ln (q)}}\right)^{2} .
\end{aligned}
$$

An application of L'Hopital's rule gives that

$$
\lim _{q \rightarrow 1^{+}} \frac{\left(e^{\alpha \sqrt{\ln (q)}}-1\right)}{\sqrt{\ln (q)}}=\alpha,
$$


which implies that in (191) we have

$$
\lim _{q \rightarrow 1^{+}}\left[\frac{\left(e^{\alpha \sqrt{\ln (q)}}-1\right)}{\sqrt{\ln (q)}}\left(1-e^{-\alpha \sqrt{\ln (q)}}\right)^{2}\right]=\alpha \cdot 0^{2}=0 .
$$

Combining (193) with (191) gives that

$$
\lim _{q \rightarrow 1^{+}}\left[\frac{1}{\sqrt{\ln (q)}} \int_{1}^{e^{\alpha \sqrt{\ln (q)}}} \frac{\left(1-\omega^{-1}\right)^{2}}{e^{(\ln \omega)^{2} / \ln (q)}} d \omega\right]=0 .
$$

We next estimate the portion of the integral in (186) over the interval $\left[e^{\alpha \sqrt{\ln (q)}}, \infty\right)$. First, since $1-\omega^{-1} \leq 1$ on this interval, one has

$$
\begin{aligned}
& \frac{1}{\sqrt{\ln (q)}} \int_{e^{\alpha} \sqrt{\ln (q)}}^{\infty} \frac{\left(1-\omega^{-1}\right)^{2}}{e^{(\ln \omega)^{2} / \ln (q)}} d \omega \\
& \leq \frac{1}{\sqrt{\ln (q)}} \int_{e^{\alpha \sqrt{\ln (q)}}}^{\infty} \frac{1}{e^{(\ln \omega)^{2} / \ln (q)}} d \omega .
\end{aligned}
$$

We next bound the right-hand side of (195), using the following estimate from $[4,14]$ :

$$
\int_{C}^{\infty} e^{-A[\ln \omega+B]^{2}} d \omega \leq \frac{\sqrt{\pi}}{2} \frac{e^{1 /(4 A)-B}}{\sqrt{A}} e^{-A[\ln (C)+B-1 /(2 A)]^{2}},
$$

which holds for $C>e^{-B+1 /(2 A)}$. Setting $A=1 / \ln (q), B=0$, and $C=e^{\alpha \sqrt{\ln (q)}}$ as in (195) yields that

$$
\begin{aligned}
& \frac{1}{\sqrt{\ln (q)}} \int_{e^{\alpha} \sqrt{\ln (q)}}^{\infty} \frac{1}{e^{(\ln \omega)^{2} / \ln (q)}} d \omega \\
& \leq \frac{1}{\sqrt{\ln (q)}} \frac{\sqrt{\pi}}{2} \frac{e^{\ln (q) / 4}}{1 / \sqrt{\ln (q)}} e^{-[\alpha \sqrt{\ln (q)}-\ln (q) / 2]^{2} / \ln (q)} \\
& \quad=\frac{\sqrt{\pi}}{2} e^{-\alpha^{2}}\left[e^{\alpha \sqrt{\ln (q)}}\right]
\end{aligned}
$$

for $e^{\alpha \sqrt{\ln (q)}}>e^{\ln (q) / 2}$, or equivalently for $1<q<e^{4 \alpha^{2}}$. Since the final expression in (197) approaches $\sqrt{\pi} e^{-\alpha^{2}} / 2$ as $q \rightarrow 1^{+}$, by choosing $\alpha$ sufficiently large one can make (197) and hence (195), arbitrarily small for $q$ and sufficiently close to 1 .

Now, let $\varepsilon>0$ be given. By (186), we have for all $\alpha>0$

$$
\begin{aligned}
0< & \widetilde{P}_{0}(q ; 0)-1 \leq F(q)\left[\frac{1}{\sqrt{\ln (q)}} \int_{1}^{e^{\alpha \sqrt{\ln (q)}}} \frac{\left(1-\omega^{-1}\right)^{2}}{e^{(\ln \omega)^{2} / \ln (q)}} d \omega\right] \\
& +F(q)\left[\frac{1}{\sqrt{\ln (q)}} \int_{e^{\alpha \sqrt{\ln (q)}}}^{\infty} \frac{\left(1-\omega^{-1}\right)^{2}}{e^{(\ln \omega)^{2} / \ln (q)}} d \omega\right] .
\end{aligned}
$$

One has from (188) and (197) that

$$
\lim _{q \rightarrow 1+}\left\{F(q) \frac{\sqrt{\pi}}{2} e^{-\alpha^{2}}\left[e^{\alpha \sqrt{\ln (q)}}\right]\right\}=\frac{1}{2} e^{-\alpha^{2}}<e^{-\alpha^{2}} .
$$

Fix $\alpha>0$ such that $e^{-\alpha^{2}}<\varepsilon / 2$. By (197) and (200), there exists $q_{1}=q_{1}(\alpha, \varepsilon)$ such that for all $1<q<q_{1}$ one has

$$
\begin{aligned}
0 & <F(q)\left[\frac{1}{\sqrt{\ln (q)}} \int_{e^{\alpha \sqrt{\ln (q)}}}^{\infty} \frac{\left(1-\omega^{-1}\right)^{2}}{e^{(\ln \omega)^{2} / \ln (q)}} d \omega\right] \\
& \leq F(q) \frac{\sqrt{\pi}}{2} e^{-\alpha^{2}}\left[e^{\alpha \sqrt{\ln (q)}}\right]<e^{-\alpha^{2}}<\frac{\varepsilon}{2} .
\end{aligned}
$$

For this value of $\alpha$, by virtue of (194), one has

$$
\lim _{q \rightarrow 1^{+}} F(q)\left[\frac{1}{\sqrt{\ln (q)}} \int_{1}^{e^{\alpha \sqrt{\ln (q)}}} \frac{\left(1-\omega^{-1}\right)^{2}}{e^{(\ln \omega)^{2} / \ln (q)}} d \omega\right]=0
$$

which in turn says that there is a $q_{2}=q_{2}(\alpha, \varepsilon)$ such that for all $1<q<q_{2}$ one has

$$
0<F(q)\left[\frac{1}{\sqrt{\ln (q)}} \int_{1}^{e^{\alpha \sqrt{\ln (q)}}} \frac{\left(1-\omega^{-1}\right)^{2}}{e^{(\ln \omega)^{2} / \ln (q)}} d \omega\right]<\frac{\varepsilon}{2}
$$

Thus, for $Q(\alpha, \varepsilon) \equiv \min \left\{q_{1}, q_{2}\right\}$ and for all $1<q<Q(\alpha, \varepsilon)$, applying (204) and (202) to (198) and (199) yields

$$
0<\widetilde{P}_{0}(q ; 0)-1<\frac{\varepsilon}{2}+\frac{\varepsilon}{2}<\varepsilon
$$

This gives the proposition.

\section{Convergence in $\mathscr{L}^{p}(\mathbb{R})$}

We turn next to convergence in $\mathscr{L}^{p}(\mathbb{R})$.

Theorem 21. $\widetilde{P}_{n}(q ; \omega)$ converges to $P_{n}(\omega) \widetilde{\chi}_{[-1,1]}(\omega)$ in $\mathscr{L}^{p}(\mathbb{R})$ as $q \rightarrow 1^{+}$for each $1 \leq p<\infty$. That is,

$$
\lim _{q \rightarrow 1^{+}}\left\|\widetilde{P}_{n}(q ; \omega)-P_{n}(\omega) \tilde{\chi}_{[-1,1]}(\omega)\right\|_{p}=0
$$

Proof. We first handle the case $p=1$, which, by boundedness of the functions under study, turns out to be sufficient to handle the remaining cases $1<p<\infty$.

Let $\varepsilon_{1}>0$ be given. First note that, by (118) and (120), there is a $Q_{1}>0$ such that

$$
0<\widetilde{P}_{0}(q ; \omega) \leq \widetilde{P}_{0}(q ; 0)<1+\varepsilon_{1}
$$


for all $1<q<Q_{1}$. Thus, $\widetilde{P}_{0}(q ; \omega)$ is uniformly bounded for $q$. Next, note that, for $\omega$ with $|\omega| \leq 2$ and $1<q<Q_{1}$, one has from (109) that

$$
\begin{aligned}
& \left|\widetilde{P}_{n}(q ; \omega)\right| \\
& \quad \leq \sum_{k=0}^{\lfloor n / 2\rfloor}\left|C_{n, n-2 k}\right||\omega|^{n-2 k} q^{k(k+1)}\left|\widetilde{P}_{0}\left(q ; \frac{\omega}{q^{k}}\right)\right| \\
& \quad \leq \sum_{k=0}^{\lfloor n / 2\rfloor}\left|C_{n, n-2 k}\right| 2^{n} q^{\lfloor n / 2\rfloor(\lfloor n / 2\rfloor+1)}\left(1+\varepsilon_{1}\right) \\
& \quad=A_{n} 2^{n} Q_{1}^{\lfloor n / 2\rfloor(\lfloor n / 2\rfloor+1)}\left(1+\varepsilon_{1}\right) \equiv B_{n},
\end{aligned}
$$

where $A_{n}=\sum_{k=0}^{\lfloor n / 2\rfloor}\left|C_{n, n-2 k}\right|$. Observe that, since $A_{n}, 2$, and $Q_{1}$ are all greater than 1 in (209), one has $B_{n}>\varepsilon_{1}$. Note also that, from (141), one has the existence of a $q_{5}>1$ such that for all $1<q<q_{5}$

$$
\left|\widetilde{P}_{n}(q ; \omega)\right|<\varepsilon_{1}
$$

for all $\omega$ such that $|\omega| \geq 3 / 2$. From (209) and (210) and the fact that $B_{n}>\varepsilon_{1}$, one has that, for $1<q<q_{6} \equiv \min \left\{Q_{1}, q_{5}\right\}$, we have

$$
\left|\widetilde{P}_{n}(q ; \omega)\right| \leq B_{n}
$$

for all $\omega \in \mathbb{R}$. Thus, $\widetilde{P}_{n}(q ; \omega)$ is uniformly bounded in $1<$ $q<q_{6}$. Letting $D_{n}=\sup \left\{\left|P_{n}(\omega)\right|:|\omega| \leq 1\right\}$, we also have that $\left|P_{n}(\omega) \widetilde{\chi}_{[-1,1]}(\omega)\right| \leq D_{n}$. Thus, our two functions of interest are bounded on $\mathbb{R}$ for $1<q<q_{6}$.

Next, choose $0<\varepsilon<1$ with $\varepsilon<\varepsilon_{1} /\left[12\left(B_{n}+D_{n}\right)\right]$. Then, by oddness/evenness, respectively, one has

$$
\begin{aligned}
\int_{-\infty}^{\infty}\left|\widetilde{P}_{n}(q ; \omega)-P_{n}(\omega) \tilde{\chi}_{[-1,1]}(\omega)\right| d \omega \\
=2 \int_{0}^{\infty}\left|\widetilde{P}_{n}(q ; \omega)-P_{n}(\omega) \tilde{\chi}_{[-1,1]}(\omega)\right| d \omega \\
=2\left\{\int_{0}^{1-\varepsilon}\left|\widetilde{P}_{n}(q ; \omega)-P_{n}(\omega) \tilde{\chi}_{[-1,1]}(\omega)\right| d \omega\right. \\
\left.+\int_{1-\varepsilon}^{1+\varepsilon}\left|\widetilde{P}_{n}(q ; \omega)-P_{n}(\omega) \tilde{\chi}_{[-1,1]}(\omega)\right| d \omega\right\} \\
+2\left\{\int_{1+\varepsilon}^{\infty}\left|\widetilde{P}_{n}(q ; \omega)-0\right| d \omega\right\},
\end{aligned}
$$

and we proceed to bound the three resulting integrals in (213) and (214)

From (130), there is a $Q_{4}>1$ such that one obtains $\left|\widetilde{P}_{n}(q ; \omega)-P_{n}(\omega) \widetilde{\chi}_{[-1,1]}(\omega)\right|<\varepsilon_{1} / 6$ for all $\omega \in[0,1-\varepsilon]$ and $1<q<Q_{4}$. This gives

$$
\begin{array}{r}
2 \int_{0}^{1-\varepsilon}\left|\widetilde{P}_{n}(q ; \omega)-P_{n}(\omega) \widetilde{\chi}_{[-1,1]}(\omega)\right| d \omega \\
\quad \leq 2 \int_{0}^{1-\varepsilon} \frac{\varepsilon_{1}}{6} d \omega=2(1-\varepsilon) \frac{\varepsilon_{1}}{6}<\frac{\varepsilon_{1}}{3}
\end{array}
$$

for all $1<q<Q_{4}$.
Next, by choice of $\varepsilon$, one has

$$
\begin{aligned}
2 \int_{1-\varepsilon}^{1+\varepsilon} & \left|\widetilde{P}_{n}(q ; \omega)-P_{n}(\omega) \tilde{\chi}_{[-1,1]}(\omega)\right| d \omega \\
& \leq 2 \int_{1-\varepsilon}^{1+\varepsilon}\left|\widetilde{P}_{n}(q ; \omega)\right|+\left|P_{n}(\omega) \tilde{\chi}_{[-1,1]}(\omega)\right| d \omega \\
& \leq 2 \int_{1-\varepsilon}^{1+\varepsilon} B_{n}+D_{n} d \omega=2(2 \varepsilon)\left(B_{n}+D_{n}\right) \\
& <2\left(2 \frac{\varepsilon_{1}}{12\left(B_{n}+D_{n}\right)}\right)\left(B_{n}+D_{n}\right)=\frac{\varepsilon_{1}}{3}
\end{aligned}
$$

for all $1<q<Q_{1}$.

Finally, from (134), one has the existence of a $q_{3}>1$ such that

$$
\begin{aligned}
2 \int_{1+\varepsilon}^{\infty}\left|\widetilde{P}_{n}(q ; \omega)-0\right| d \omega \\
\leq 2\left[\frac{2\left(\mu_{q^{2}}\right)^{3} \ln (q)}{\pi f_{0, q}(0)}\right]\{\sqrt{\pi}-\sqrt{\ln (q)}\}^{-1} \\
\times \sqrt{\frac{\pi}{2}} q^{n(n+2) / 4} A_{n} \int_{1+\varepsilon}^{\infty} e^{-[\ln (|\omega|)-(n+1) \ln (q) / 2]^{2} / \ln (q)} d \omega
\end{aligned}
$$

for all $1<q<q_{3}$. Now, from (151), one has

$$
\begin{aligned}
\int_{1+\varepsilon}^{\infty} & e^{-[\ln (|\omega|)-(n+1) \ln (q) / 2]^{2} / \ln (q)} d \omega \\
\leq & \frac{\sqrt{\pi}}{2} \sqrt{\ln (q)} e^{\ln (q) / 4+(n+1) \ln (q) / 2} \\
& \times e^{-[\ln (1+\varepsilon)-(n+1) \ln (q) / 2-\ln (q) / 2]^{2} / \ln (q)} \\
= & \frac{\sqrt{\pi}}{2} \sqrt{\ln (q)} q^{1 / 4} q^{(n+1) / 2} \\
& \times e^{-[\ln (1+\varepsilon)-(n+2) \ln (q) / 2]^{2} / \ln (q)}
\end{aligned}
$$

for $1+\varepsilon>e^{(n+2) \ln (q) / 2}=q^{(n+2) / 2}$. Thus, one uses (221) to bound (219) for $1<q<(1+\varepsilon)^{2 /(n+2)}$, obtaining

$$
\begin{aligned}
2 \int_{1+\varepsilon}^{\infty} \mid \widetilde{P}_{n} & (q ; \omega)-0 \mid d \omega \\
\leq & 2\left[\frac{2\left(\mu_{q^{2}}\right)^{3} \ln (q)}{\pi f_{0, q}(0)}\right]\{\sqrt{\pi}-\sqrt{\ln (q)}\}^{-1} \\
& \times \frac{\pi A_{n}}{2 \sqrt{2}} \sqrt{\ln (q)} q^{(n+1)(n+3) / 4} \\
& \times e^{-[\ln (1+\varepsilon)-(n+2) \ln (q) / 2]^{2} / \ln (q)} .
\end{aligned}
$$

Now, from (17), we have that (223) approaches 0 as $q \rightarrow 1^{+}$. Thus, there is a $Q_{5}>1$ such that, for $1<q<Q_{5}$,

$$
2 \int_{1+\varepsilon}^{\infty}\left|\widetilde{P}_{n}(q ; \omega)-0\right| d \omega \leq \frac{\varepsilon_{1}}{3} .
$$


Finally, using (215), (217), and (224) to bound (213) and (214) gives that

$$
\begin{aligned}
& \left\|\widetilde{P}_{n}(q ; \omega)-P_{n}(\omega) \tilde{\chi}_{[-1,1]}(\omega)\right\|_{1} \\
& \quad=\int_{-\infty}^{\infty}\left|\widetilde{P}_{n}(q ; \omega)-P_{n}(\omega) \tilde{\chi}_{[-1,1]}(\omega)\right| d \omega \\
& \quad \leq \frac{\varepsilon_{1}}{3}+\frac{\varepsilon_{1}}{3}+\frac{\varepsilon_{1}}{3}=\varepsilon_{1}
\end{aligned}
$$

for all $1<q<\min \left\{1+\varepsilon, q_{3}, q_{6}, Q_{1}, Q_{4}, Q_{5}\right\}$. Thus, (206) holds in the case $p=1$.

Next, observe that if $f$ and $g$ are both bounded on $\mathbb{R}$, then, for $1 \leq p<\infty$, one has

$$
\begin{aligned}
& \int_{-\infty}^{\infty}|f(x)-g(x)|^{p} d x \\
& \quad=\int_{-\infty}^{\infty}|f(x)-g(x)|^{p-1}|f(x)-g(x)| d x \\
& \quad \leq\|f-g\|_{\infty}^{p-1} \int_{-\infty}^{\infty}|f(x)-g(x)| d x \\
& \quad=\|f-g\|_{\infty}^{p-1}\|f-g\|_{1} .
\end{aligned}
$$

Thus, in this bounded setting, $\mathscr{L}^{1}$ convergence is sufficient for $\mathscr{L}^{p}$ convergence.

We have observed that $\left|\widetilde{P}_{n}(q ; \omega)\right|$ is bounded by $B_{n}$ for $1<$ $q<q_{6}$. Also $\left|P_{n}(\omega) \tilde{\chi}_{[-1,1]}(\omega)\right| \leq D_{n}$. By the above remarks, it follows that (206) now also holds in the cases $1<p<\infty$, and the theorem is proven.

\section{Approximating $\widetilde{P}_{n}(q ; \omega)$}

The goal of this section is to provide and analyze the two approximations for $\widetilde{P}_{n}(q ; \omega)$ given by (228) and (229) below. These two expressions are related to the exact expression (109) for $\widetilde{P}_{n}(q ; \omega)$, but they are computationally simpler approximations of $\widetilde{P}_{n}(q ; \omega)$. We will also show that as $q \rightarrow 1^{+}$ the difference of $\widetilde{P}_{n}(q ; \omega)$ with each of the approximations (228) and (229) is converging uniformly to 0 on compact sets of $\mathbb{R}$.

The first expression, (228), is obtained by removing all delays in (109):

$$
\widetilde{P}_{n}(q ; \omega) \approx \widetilde{P}_{0}(q ; \omega) \sum_{k=0}^{\lfloor n / 2\rfloor} C_{n, n-2 k} \omega^{n-2 k} q^{k(k+1)}
$$

The second expression, (229), is obtained by setting $q=1$ in the summation for (228):

$$
\widetilde{P}_{n}(q ; \omega) \approx \widetilde{P}_{0}(q ; \omega) P_{n}(\omega)=\widetilde{P}_{0}(q ; \omega) \sum_{k=0}^{\lfloor n / 2\rfloor} C_{n, n-2 k} \omega^{n-2 k}
$$

The convergence is as follows. Given any compact set $C \subset \mathbb{R}$, there is an $\omega_{0}$ with $C \subset\left[-\omega_{0}, \omega_{0}\right] \subset \mathbb{R}$. Given such $C$ and corresponding $\omega_{0}$ and given $\varepsilon>0$, there is a $\widehat{q}>1$ such that for all $1<q<\widehat{q}$ one has

$$
\begin{aligned}
& \left|\widetilde{P}_{n}(q ; \omega)-\widetilde{P}_{0}(q ; \omega) \sum_{k=0}^{\lfloor n / 2\rfloor} C_{n, n-2 k} \omega^{n-2 k} q^{k(k+1)}\right|<\varepsilon, \\
& \left|\widetilde{P}_{n}(q ; \omega)-\widetilde{P}_{0}(q ; \omega) \sum_{k=0}^{\lfloor n / 2\rfloor} C_{n, n-2 k} \omega^{n-2 k}\right|<\varepsilon,
\end{aligned}
$$

for all $\omega \in\left[-\omega_{0}, \omega_{0}\right]$ and therefore for all $\omega \in C$. Note first that, for $n=0,1$ there is equality in (228) and (229) for all $q>1$ and all $\omega \in \mathbb{R}$. So, we assume that $n \geq 2$ and proceed as follows.

To obtain convergence for (228), note first that, for each $p \geq 0$, one has

$$
\begin{aligned}
\left|\widetilde{P}_{0}(q ; \omega)-\widetilde{P}_{0}\left(q ; \frac{\omega}{q^{p}}\right)\right| \\
=\frac{2\left(\mu_{q^{2}}\right)^{3}}{\pi f_{0, q}(0)} \\
\quad \times\left|\int_{-\infty}^{\omega} \frac{v}{\theta\left(q^{2} ; v^{2}\right)} d v-\int_{-\infty}^{\omega / q^{p}} \frac{v}{\theta\left(q^{2} ; v^{2}\right)} d v\right| \\
=\frac{2\left(\mu_{q^{2}}\right)^{3}}{\pi f_{0, q}(0)}\left|\int_{\omega / q^{p}}^{\omega} \frac{v}{\theta\left(q^{2} ; v^{2}\right)} d v\right| \\
=\frac{2\left(\mu_{q^{2}}\right)^{3}}{\pi f_{0, q^{\prime}}(0)} \int_{|\omega| / q^{p}}^{|\omega|} \frac{v}{\theta\left(q^{2} ; v^{2}\right)} d v \\
\leq \frac{2\left(\mu_{q^{2}}\right)^{3}}{\pi f_{0, q}(0)}\left\{|\omega|-\frac{|\omega|}{q^{p}}\right\} \max \left(\frac{v}{\theta\left(q^{2} ; v^{2}\right)}\right) \\
=\frac{2\left(\mu_{q^{2}}\right)^{3}}{\pi f_{0, q}(0)}|\omega|\left\{1-\frac{1}{q^{p}}\right\} \frac{1}{\theta\left(q^{2} ; 1^{2}\right)},
\end{aligned}
$$

where the maximum of $v / \theta\left(q^{2} ; v^{2}\right)$ occurring at 1 is shown in [4]. From (26), we bound $1 / \theta\left(q^{2} ; 1^{2}\right)$ in (232) from the above to give

$$
\begin{aligned}
& \left|\widetilde{P}_{0}(q ; \omega)-\widetilde{P}_{0}\left(q ; \frac{\omega}{q^{p}}\right)\right| \\
& \quad \leq \frac{2\left(\mu_{q^{2}}\right)^{3}}{\pi f_{0, q}(0)}|\omega|\left\{1-\frac{1}{q^{p}}\right\} \frac{q^{-1 / 4}}{\sqrt{\pi / \ln (q)}-1} \\
& \quad=\frac{2 \ln (q)\left(\mu_{q^{2}}\right)^{3}}{\pi f_{0, q}(0)} \frac{1}{\sqrt{\ln (q)}|\omega|\left\{1-\frac{1}{q^{p}}\right\} \frac{q^{-1 / 4}}{\sqrt{\pi}-\sqrt{\ln (q)}}} \\
& \quad=\frac{2 \ln (q)\left(\mu_{q^{2}}\right)^{3}}{\pi f_{0, q}(0)}|\omega| \frac{(q-1)}{\sqrt{\ln (q)}}\left[\frac{\sum_{k=0}^{p-1} q^{k}}{q^{p}}\right]
\end{aligned}
$$




$$
\begin{aligned}
& \times \frac{q^{-1 / 4}}{\sqrt{\pi}-\sqrt{\ln (q)}} \\
\leq & \frac{2 \ln (q)\left(\mu_{q^{2}}\right)^{3}}{\pi f_{0, q}(0)}\left|\omega_{0}\right| \frac{(q-1)}{\ln (q)} \sqrt{\ln (q)}[p] \frac{q^{-1 / 4}}{\sqrt{\pi}}
\end{aligned}
$$

for all $\omega$ with $|\omega| \leq\left|\omega_{0}\right|$.

From L'Hopital's rule, one has that

$$
\lim _{q \rightarrow 1^{+}} \frac{q-1}{\ln (q)}=1
$$

which combines with (138) to imply that the coefficients in (234) have the following limit:

$$
\begin{aligned}
\lim _{q \rightarrow 1^{+}} \frac{2 \ln (q)\left(\mu_{q^{2}}\right)^{3}}{\pi f_{0, q}(0)} \frac{q-1}{\ln (q)} \frac{q^{-1 / 4}}{\sqrt{\pi}} & =\frac{2}{\pi} \cdot \frac{\pi}{2} \cdot 1 \cdot \frac{1}{\sqrt{\pi}} \\
& =\frac{1}{\sqrt{\pi}}<1 .
\end{aligned}
$$

Thus, from (234) and (236), one has that there is a $q_{7}$ such that, for all $1<q<q_{7}$ and all $|\omega| \leq\left|\omega_{0}\right|$,

$$
\left|\widetilde{P}_{0}(q ; \omega)-\widetilde{P}_{0}\left(q ; \frac{\omega}{q^{p}}\right)\right| \leq 1 \cdot\left|\omega_{0}\right| p \sqrt{\ln (q)} .
$$

Thus, for $0 \leq k \leq p$, one has

$$
\left|\widetilde{P}_{0}(q ; \omega)-\widetilde{P}_{0}\left(q ; \frac{\omega}{q^{k}}\right)\right| \leq\left|\omega_{0}\right| k \sqrt{\ln (q)} \leq\left|\omega_{0}\right| p \sqrt{\ln (q)}
$$

for all $|\omega| \leq\left|\omega_{0}\right|$ and all $1<q<q_{7}$.

Now, from (109) coupled with (238), one has that

$$
\begin{aligned}
& \left|\widetilde{P}_{n}(q ; \omega)-\widetilde{P}_{0}(q ; \omega) \sum_{k=0}^{\lfloor n / 2\rfloor} C_{n, n-2 k} \omega^{n-2 k} q^{k(k+1)}\right| \\
& \quad=\left|\sum_{k=0}^{\lfloor n / 2\rfloor} C_{n, n-2 k} \omega^{n-2 k} q^{k(k+1)}\left(\widetilde{P}_{0}\left(q ; \frac{\omega}{q^{k}}\right)-\widetilde{P}_{0}(q ; \omega)\right)\right| \\
& \quad \leq\left(\sum_{k=0}^{\lfloor n / 2\rfloor}\left|C_{n, n-2 k}\right|\left|\omega_{0}\right|^{n-2 k} q_{7}^{k(k+1)}\right)\left|\omega_{0}\right|\left\lfloor\frac{n}{2}\right\rfloor \sqrt{\ln (q)}
\end{aligned}
$$

for all $|\omega| \leq\left|\omega_{0}\right|$ and all $1<q<q_{7}$. Placing (240) below $\varepsilon$ and solving the resulting inequality for $q$ gives that, given $\varepsilon>0$, there is a $q_{\varepsilon}>1$ given by

$$
\begin{aligned}
& q_{\varepsilon} \min \left\{q_{7}, \exp \left(\varepsilon^{2}[\right.\right. \\
&\left.\qquad \sum_{k=0}^{\lfloor n / 2\rfloor}\left|C_{n, n-2 k}\right|\left|\omega_{0}\right|^{n-2 k} q_{7}^{k(k+1)}\right)^{2} \\
&\left.\left.\times\left|\omega_{0}\right|^{2}\left\lfloor\left.\frac{n}{2}\right|^{2}\right]^{-1}\right)\right\}
\end{aligned}
$$

such that

$$
\left|\widetilde{P}_{n}(q ; \omega)-\widetilde{P}_{0}(q ; \omega) \sum_{k=0}^{\lfloor n / 2\rfloor} C_{n, n-2 k} \omega^{n-2 k} q^{k(k+1)}\right|<\varepsilon
$$

for all $1<q<q_{\varepsilon}$ and $\omega \in\left[-\omega_{0}, \omega_{0}\right]$. This gives convergence for (228).

We turn next to (229). Observe that, for $|\omega| \leq\left|\omega_{0}\right|$, one has

$$
\begin{aligned}
& \mid \widetilde{P}_{0}(q ; \omega) \sum_{k=0}^{\lfloor n / 2\rfloor} C_{n, n-2 k} \omega^{n-2 k} \\
& -\widetilde{P}_{0}(q ; \omega) \sum_{k=0}^{\lfloor n / 2\rfloor} C_{n, n-2 k} \omega^{n-2 k} q^{k(k+1)} \mid \\
& \quad=\left|\widetilde{P}_{0}(q ; \omega) \sum_{k=0}^{\lfloor n / 2\rfloor} C_{n, n-2 k} \omega^{n-2 k}\left(1-q^{k(k+1)}\right)\right| \\
& \leq \max \left\{\left|\widetilde{P}_{0}(q ; \omega)\right|\right\} \sum_{k=0}^{\lfloor n / 2\rfloor}\left|C_{n, n-2 k}\right|\left|\omega_{0}\right|^{n-2 k}\left(q^{k(k+1)}-1\right) \\
& \quad \leq \widetilde{P}_{0}(q ; 0) \cdot \sum_{k=0}^{\lfloor n / 2\rfloor}\left|C_{n, n-2 k}\right|\left|\omega_{0}\right|^{n-2 k}\left(q^{\lfloor n / 2\rfloor(\lfloor n / 2\rfloor+1)}-1\right) \\
& \quad \leq 2 \cdot \sum_{k=0}^{\lfloor n / 2\rfloor}\left|C_{n, n-2 k}\right|\left|\omega_{0}\right|^{n-2 k}\left(q^{\lfloor n / 2\rfloor(\lfloor n / 2\rfloor+1)}-1\right)
\end{aligned}
$$

where (244) follows from (118) and (245) holds for $1<q<$ $Q$ with $Q$ chosen as in Proposition 20 (for $\varepsilon$ set equal to 1 ). Placing (245) below $\varepsilon$ and solving the resulting inequality for $q$ gives that, given $\varepsilon>0$, there is a $\widetilde{q}_{\varepsilon}>1$, given by

$\widetilde{q}_{\varepsilon}$

$$
=\min \left\{Q,\left(1+\frac{\varepsilon}{2 \sum_{k=0}^{\lfloor n / 2\rfloor}\left|C_{n, n-2 k}\right|\left|\omega_{0}\right|^{n-2 k}}\right)^{1 /\{\lfloor n / 2\rfloor(\lfloor n / 2\rfloor+1)\}}\right\}
$$

such that

$$
\begin{aligned}
& \mid \widetilde{P}_{0}(q ; \omega) \sum_{k=0}^{\lfloor n / 2\rfloor} C_{n, n-2 k} \omega^{n-2 k} \\
& -\widetilde{P}_{0}(q ; \omega) \sum_{k=0}^{\lfloor n / 2\rfloor} C_{n, n-2 k} \omega^{n-2 k} q^{k(k+1)} \mid<\varepsilon
\end{aligned}
$$

for $|\omega| \leq\left|\omega_{0}\right|$ and $1<q<\widehat{q}_{\varepsilon}$. Thus, by setting $\varepsilon=\widetilde{\varepsilon} / 2$ in (242) and (247) and relying on the triangle inequality, one has

$$
\begin{aligned}
& \left|\widetilde{P}_{n}(q ; \omega)-\widetilde{P}_{0}(q ; \omega) P_{n}(\omega)\right| \\
& \quad \leq\left|\widetilde{P}_{n}(q ; \omega)-\widetilde{P}_{0}(q ; \omega) \sum_{k=0}^{\lfloor n / 2\rfloor} C_{n, n-2 k} \omega^{n-2 k} q^{k(k+1)}\right|
\end{aligned}
$$




$$
\begin{aligned}
& +\mid \widetilde{P}_{0}(q ; \omega) \sum_{k=0}^{\lfloor n / 2\rfloor} C_{n, n-2 k} \omega^{n-2 k} q^{k(k+1)} \\
& \quad-\widetilde{P}_{0}(q ; \omega) \sum_{k=0}^{\lfloor n / 2\rfloor} C_{n, n-2 k} \omega^{n-2 k} \mid \\
& \leq \frac{\widetilde{\varepsilon}}{2}+\frac{\widetilde{\varepsilon}}{2}=\widetilde{\varepsilon}
\end{aligned}
$$

for $|\omega| \leq\left|\omega_{0}\right|$ and $1<q<\min \left\{q_{\tilde{\varepsilon} / 2}, \widehat{q}_{\tilde{\varepsilon} / 2}\right\}$. This gives convergence for (229). Now let $\widehat{q}=\min \left\{q_{\varepsilon}, q_{\widetilde{\varepsilon} / 2}, \widehat{q}_{\widetilde{\varepsilon} / 2}\right\}$ and the result holds.

We remark here that $\widetilde{P}_{0}(q ; \omega)$ in $(229)$ is a smooth Schwartz analogue of the truncation function $\widetilde{\chi}_{[-1,1]}(\omega)$ in (19). Note also that the approximations (228) and (229) need not be wavelets, while each $\widetilde{P}_{n}(q ; \omega)$ for $n \geq 1$ is a wavelet. Furthermore, neither approximation (228) nor (229) satisfies any naturally apparent differential equation. Thus, one may be giving up useful properties if one relies on the somewhat simpler approximations (228) or (229) in place of (109).

\section{Remaining a MADE under Inverse Fourier Transform}

In this section, the goal is to give a generalization of the techniques used in Section 4. This generalization gives a condition on an original MADE sufficient to conclude that its resulting inverse Fourier transform remains a MADE. This motivates the search for a global solution for a MADE with 0 value at $\omega=0$, because its inverse Fourier transform will be a wavelet solution to a corresponding MADE.

Consider a differential equation of the form

$$
a\left(t, D_{t}\right) f(t)=\left[b\left(\frac{t}{q}, D_{t}\right) f\right](q t) \text {. }
$$

Here $a(t, \tau)$ and $b(t / q, \tau)$ are polynomials in the two variables $t / q$ and $\tau$ as in (250). More explicitly, (249) is given in expanded form by

$$
\sum a_{i j} t^{i} D_{t}^{j} f(t)=\sum b_{m k} t^{m} \cdot\left[D_{t}^{k} f\right](q t)
$$

Now, suppose that there are nonnegative integers $N_{a}$ and $M_{b}$ so that as $t \rightarrow 0$

$$
\begin{aligned}
a(t, \tau) & =\sum a_{i j} t^{i} \tau^{j} \longrightarrow \sum_{j=0}^{N_{a}} a_{0 j} \tau^{j} \equiv A(\tau), \\
b\left(\frac{t}{q}, \tau\right) & =\sum b_{m k}\left(\frac{t}{q}\right)^{m} \tau^{k} \longrightarrow \sum_{k=0}^{M_{b}} b_{0 k} \tau^{k} \equiv B(\tau),
\end{aligned}
$$

where the degrees $\operatorname{deg} A(\tau)$ and $\operatorname{deg} B(\tau)$ are not both $-\infty$ (i.e., $a_{0 N_{a}} \neq 0$ or $b_{0 M_{b}} \neq 0$ ). Then, letting $t \rightarrow 0$ in the operators in (249) (but not in the arguments for the function $f)$, one obtains $A(\tau) f(t)=[B(\tau) f](q t)$ or equivalently
$A\left(D_{t}\right) f(t)=\left[B\left(D_{t}\right) f\right](q t)$. That is, as $t \rightarrow 0$ in the operators, (249) and (250) formally converge to the equation

$$
\begin{aligned}
& a_{0 N_{a}}\left[\tau^{N_{a}} f\right](t)+\sum_{j=0}^{N_{a}-1} a_{0 j}\left[\tau^{j} f\right](t) \\
& \quad=b_{0 M_{b}}\left[\tau^{M_{b}} f\right](q t)+\sum_{k=0}^{M_{b}-1} b_{0 k}\left[\tau^{k} f\right](q t),
\end{aligned}
$$

which is equivalent to

$$
\begin{aligned}
& a_{0 N_{a}}\left[D_{t}^{N_{a}} f\right](t)+\sum_{j=0}^{N_{a}-1} a_{0 j}\left[D_{t}^{j} f\right](t) \\
& =b_{0 M_{b}}\left[D_{t}^{M_{b}} f\right](q t)+\sum_{k=0}^{M_{b}-1} b_{0 k}\left[D_{t}^{k} f\right](q t) .
\end{aligned}
$$

We refer to (249) as a MADE if it formally converges to (253) where $\operatorname{deg} B(\tau) \leq \operatorname{deg} A(\tau)$ as $t \rightarrow 0$ (i.e., $M_{b} \leq N_{a}$ ). In other words, (249) is said to be a MADE when the terms with the highest order derivative having constant coefficients in (249) have one term with argument $t$ of $f$ not multiplicatively advanced or delayed by $q$ and some lower or equal order term with constant coefficient does contain an argument of form $q t$.

Next, take the inverse Fourier transform of (250) and denote $\mathscr{F}^{-1}[f]=\widehat{f}$. Then, from (46) and (58), one obtains

$$
a\left(-i D_{\omega},-i \omega\right) \widehat{f}(\omega)=\left(\frac{1}{q}\right) b\left(-i D_{\omega},-\frac{i \omega}{q}\right) \widehat{f}\left(\frac{\omega}{q}\right),
$$

where the polynomials $a$ and $b$ are identical to those in (249). Now, using the derivation property of $D_{\omega}$ to reorder the $\omega$ terms to precede the $D_{\omega}$ terms in (254), collecting powers of $i$, and absorbing powers of $q$ into coefficients, one obtains two resulting polynomials $\widehat{a}(\omega, w)$ and $\widehat{b}(q \omega, w)$ in the two variables $\omega$ and $w$ with

$$
\widehat{a}\left(\omega, D_{\omega}\right) \widehat{f}(\omega)=\left[\widehat{b}\left(q \omega, D_{\omega}\right) \widehat{f}\right]\left(\frac{\omega}{q}\right) .
$$

Now, suppose that as $\omega \rightarrow 0$ in the operators of (255) one obtains

$$
\begin{aligned}
& \widehat{a}(\omega, w) \longrightarrow \sum_{j=0}^{\widehat{N}_{a}} \widehat{a}_{j} w^{j} \equiv \widehat{A}(w), \\
& \widehat{b}(\omega, w) \longrightarrow \sum_{k=0}^{\widehat{M}_{b}} \widehat{b}_{k} w^{k} \equiv \widehat{B}(w),
\end{aligned}
$$

where $\operatorname{deg} \widehat{A}$ and $\operatorname{deg} \widehat{B}$ are not both $-\infty$. (i.e., $\widehat{a}_{\widehat{N}_{a}} \neq 0$ or $\left.\widehat{b}_{\widehat{N}_{b}} \neq 0\right)$. Then, similar to the process for (253), one has (255) that formally converges to the equation

$$
\begin{aligned}
\widehat{a}_{\widehat{N}_{\hat{a}}}\left[D_{\omega}^{\widehat{N}_{\hat{a}}} \hat{f}\right](\omega)+\sum_{j=0}^{\widehat{N}_{\hat{a}}-1} \widehat{a}_{j}\left[D_{\omega}^{j} \widehat{f}\right](\omega) \\
=\widehat{b}_{\widehat{M}_{\hat{b}}}\left[D_{\omega}^{\widehat{M}_{\hat{b}}} \hat{f}\right]\left(\frac{\omega}{q}\right)+\sum_{k=0}^{\widehat{M}_{\hat{b}}-1} \widehat{b}_{k}\left[D_{\omega}^{k} \hat{f}\right]\left(\frac{\omega}{q}\right) .
\end{aligned}
$$


Now, when $\operatorname{deg} \widehat{B} \geq \operatorname{deg} \widehat{A}$ (i.e., $\widehat{M}_{\widehat{b}} \geq \widehat{N}_{\widehat{a}}$ ), (257) will not have its highest order term with constant coefficients satisfying that its argument is neither advanced nor delayed by $q>1$. Thus, when $\operatorname{deg} \widehat{B} \geq \operatorname{deg} \widehat{A}$ (i.e., $\widehat{M}_{\widehat{b}} \geq \widehat{N}_{\widehat{a}}$ ), we change variables so that $u=\omega / q$ to give

$$
\begin{aligned}
\widehat{b}_{\widehat{M}_{\hat{b}}} & {\left[D_{u}^{\widehat{M}_{\hat{b}}} \widehat{f}\right](u)+\sum_{k=0}^{\widehat{M}_{\hat{b}}-1} \widehat{b}_{k}\left[D_{u}^{k} \widehat{f}\right](u) } \\
=q \widehat{a}_{\widehat{N}_{\widehat{a}}} & {\left[D_{u}^{\widehat{N}_{\hat{a}}} \widehat{f}\right](q u)+q \sum_{j=0}^{\widehat{N}_{\widehat{a}}-1} \widehat{a}_{j}\left[D_{u}^{j} \hat{f}\right](q u), }
\end{aligned}
$$

yielding a MADE. Similarly, one now relies on the change of variable $u=\omega / q$ to convert (255) into a MADE. Thus, (249) starts as a MADE when $\operatorname{deg} B(\tau) \leq \operatorname{deg} A(\tau)$ (i.e., $M_{b} \leq N_{a}$ ) and converts to a MADE under inverse Fourier transform when $\operatorname{deg} \widehat{B} \geq \operatorname{deg} \widehat{A}$ (i.e., $\widehat{M}_{\widehat{b}} \geq \widehat{N}_{\widehat{a}}$ ).

Example 22. Equation (57) in the proof of Theorem 6 is

$$
\begin{aligned}
& t^{2} j_{n}^{\prime \prime}(q ; t)+2 t j_{n}^{\prime}(q ; t)-n(n+1) j_{n}(q ; t) \\
& \quad=-q^{n+3} t^{2} j_{n}(q ; q t)
\end{aligned}
$$

which can be rewritten as

$$
a\left(t, D_{t}\right) j_{n}(q ; t)=\left[b\left(\frac{t}{q}, D_{t}\right) j_{n}\right](q ; q t),
$$

where

$$
\begin{aligned}
& a(t, \tau)=t^{2} \tau^{2}+2 t \tau-n(n+1), \\
& b\left(\frac{t}{q}, \tau\right)=-q^{n+3}\left(\frac{t}{q}\right)^{2} .
\end{aligned}
$$

Note that substituting $q t$ into $(t / q)^{2}$ in (260) gives $t^{2}$ in (259). For the case $n \geq 1$, taking limits as $t \rightarrow 0$ gives

$$
\begin{aligned}
& \lim _{t \rightarrow 0} a(t, \tau)=-n(n+1) \equiv A(\tau), \\
& \lim _{t \rightarrow 0} b\left(\frac{t}{q}, \tau\right)=0 \equiv B(\tau) .
\end{aligned}
$$

Thus, (259) is appropriately considered a MADE as $\operatorname{deg} A=$ $0 \geq \operatorname{deg} B=-\infty$. That is the highest order term in (259) with constant coefficients, namely, $-n(n+1) j_{n}(q ; t)$, does not have its argument $t$ advanced or delayed by $q>1$.

Taking inverse Fourier transforms of both sides of (259), relying on (46) and (58), and multiplying through by $(-i)^{n} \sqrt{2 / \pi}$ yields (64); namely,

$$
\begin{aligned}
& \omega^{2} D_{\omega}^{2} \widetilde{P}_{n}(q ; \omega)+2 \omega D_{\omega} \widetilde{P}_{n}(q ; \omega)-n(n+1) \widetilde{P}_{n}(q ; \omega) \\
& \quad=q^{n+2} D_{\omega}^{2}\left\{\widetilde{P}_{n}\left(q ; \frac{\omega}{q}\right)\right\} .
\end{aligned}
$$

This is equivalent to

$$
\widehat{a}\left(\omega, D_{\omega}\right) \widetilde{P}_{n}(q ; \omega)=\left[\widehat{b}\left(q \omega, D_{\omega}\right) \widetilde{P}_{n}\right]\left(q ; \frac{\omega}{q}\right),
$$

where

$$
\begin{aligned}
& \widehat{a}(\omega, w)=\omega^{2} w^{2}+2 \omega w-n(n+1), \\
& \widehat{b}(q \omega, w)=q^{n} w^{2} .
\end{aligned}
$$

Taking limits as $\omega \rightarrow 0$ gives

$$
\begin{aligned}
& \lim _{\omega \rightarrow 0} \widehat{a}(\omega, w)=-n(n+1) \equiv \widehat{A}(w), \\
& \lim _{\omega \rightarrow 0} \widehat{b}(\omega, w)=q^{n} w^{2} \equiv \widehat{B}(w)
\end{aligned}
$$

with $\operatorname{deg} \widehat{A}=0 \leq \operatorname{deg} \widehat{B}=2$. Since $\widehat{B}$ acts on a term with a delayed argument in (264), one makes the substitution $u=$ $\omega / q$ in (263) to obtain the MADE equivalent to (56)

$$
\begin{aligned}
(q u)^{2} & \left\{\frac{1}{q} D_{u}\right\}^{2}\left\{\widetilde{P}_{n}(q ; q u)\right\}+2(q u)\left\{\frac{1}{q} D_{u}\right\}\left\{\widetilde{P}_{n}(q ; q u)\right\} \\
& -n(n+1) \widetilde{P}_{n}(q ; q u)=q^{n+2}\left\{\frac{1}{q} D_{u}\right\}^{2} \widetilde{P}_{n}(q ; u) .
\end{aligned}
$$

Example 23. For $q>1$, consider the MADE

$$
D_{t} t D_{t}^{2} t D_{t} f(t)=f(q t) .
$$

Equation (268) is appropriately considered a MADE as follows. After using derivation properties to have the $t$ variables precede the $D_{t}$ operators, (268) becomes

$$
2 D_{t}^{2} f(t)+4 t D_{t}^{3} f(t)+t^{2} D_{t}^{4} f(t)=f(q t) .
$$

With the corresponding polynomials

$$
a(t, \tau)=2 \tau^{2}+4 t \tau^{3}+t^{2} \tau^{4}, \quad b\left(\frac{t}{q}, \tau\right)=1 .
$$

Letting $t \rightarrow 0$ yields $a(0, \tau)=2 \tau^{2}=A(\tau)$ and $b(0, \tau)=1$. Since $\operatorname{deg} A=2>\operatorname{deg} B=0$, (269) and (268) are MADEs.

Taking inverse Fourier transform of (268) and relying on (46) and (58), one obtains

$$
\begin{aligned}
(-i \omega) & \left(-i D_{\omega}\right)(-i \omega)^{2}\left(-i D_{\omega}\right)(-i \omega) \mathscr{F}^{-1}[f(t)](\omega) \\
= & \frac{1}{q} \mathscr{F}^{-1}[f(t)]\left(\frac{\omega}{q}\right),
\end{aligned}
$$

which simplifies to

$$
\omega D_{\omega} \omega^{2} D_{\omega} \omega \widehat{f}(\omega)=-q^{-1} \hat{f}\left(\frac{\omega}{q}\right) .
$$

After using derivation properties to have the $\omega$ variables precede the $D_{\omega}$ operators, (272) becomes

$$
2 \omega^{2} \widehat{f}(\omega)+4 \omega^{3} D_{\omega} \widehat{f}(\omega)+\omega^{4} D_{\omega} \widehat{f}(\omega)=-q^{-1} \widehat{f}\left(\frac{\omega}{q}\right)
$$

with the corresponding polynomials

$$
\widehat{a}(\omega, w)=2 \omega^{2}+4 \omega^{3} w+\omega^{4} w^{2}, \quad \widehat{b}(q \omega, w)=-q^{-1} .
$$


Letting $\omega \rightarrow 0$ yields $\widehat{a}(0, w)=0=\widehat{A}(w)$ and $b(0, w)=-q^{-1}$. Since $\operatorname{deg} \widehat{B}=0>\operatorname{deg} \widehat{A}=-\infty$, we convert (273) by letting $u=\omega / q$, which results in placing our equation in MADE form. For our purposes here, it will be more convenient to make this substitution in (272) rather than (273). Thus, since the highest order term with constant coefficients is $-q^{-1} \widehat{f}(\omega / q)$, we make the substitution $u=\omega / q$ with $D_{\omega}=$ $q^{-1} D_{u}$ to reexpress (272) as the MADE

$$
q u q^{-1} D_{u}(q u)^{2} q^{-1} D_{u}(q u)\{\hat{f}(q u)\}=-q^{-1} \widehat{f}(u),
$$

which simplifies to the following equation:

$$
\widehat{f}(u)=-q^{3} u D_{u} u^{2} D_{u} u\{\hat{f}(q u)\} .
$$

We exhibit a function $\widehat{f}$ with $\widehat{f}(\omega)$ a solution of (272) (and with $\widehat{f}(u)$ a solution of (276) as well) satisfying the condition that $\widehat{f}(0)=0$. Set

$$
\widehat{f}(\omega)=\frac{q^{\operatorname{Sin}(1 /[q \omega])}}{\omega} .
$$

To verify (272), one proceeds as follows:

$$
\begin{aligned}
\omega D_{\omega} \omega^{2} D_{\omega} \omega \widehat{f}(\omega) & =\omega D_{\omega} \omega^{2} D_{\omega q} \operatorname{Sin}\left(\frac{1}{q \omega}\right) \\
& =\omega D_{\omega} \omega^{2}\left(\frac{-q}{q \omega^{2}}{ }_{q} \operatorname{Cos}\left(\frac{1}{\omega}\right)\right) \\
& =-\omega D_{\omega q} \operatorname{Cos}\left(\frac{1}{\omega}\right) \\
& =\omega\left(\frac{1}{\omega^{2}}(-1) q_{q} \operatorname{Sin}\left(\frac{1}{\omega}\right)\right) \\
& =-q^{-1} \frac{q^{\operatorname{Sin}}(1 /[q(\omega / q)])}{\omega / q} \\
& =-q^{-1} \hat{f}\left(\frac{\omega}{q}\right) .
\end{aligned}
$$

We have relied on the facts that

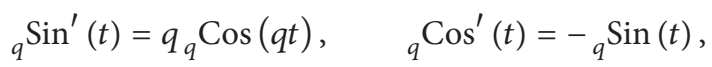

where ${ }_{q} \operatorname{Sin}(t)$ is given by (9) and ${ }_{q} \operatorname{Cos}(t)$ is given by (28). These facts are proven in [5].

Note that since $\operatorname{Sin}(\omega)$ is Schwartz, one has $\hat{f}(0)=$ $\lim _{\omega \rightarrow 0} \operatorname{Sin}(1 /[q \omega]) / \omega=0$. And thus the solution to the MADE (268) given by

$$
\begin{aligned}
f(t) & =\mathscr{F}[\widehat{f}(\omega)](t) \\
& =\frac{1}{\sqrt{2 \pi}} \int_{-\infty}^{\infty} e^{-i t \omega} \frac{q \operatorname{Sin}(1 /[q \omega])}{\omega} d \omega \\
& =\frac{1}{\sqrt{2 \pi}} \int_{-\infty}^{\infty} \cos (\omega t) \frac{q^{\operatorname{Sin}(1 /[q \omega])}}{\omega} d \omega
\end{aligned}
$$

$$
\begin{aligned}
& =\frac{-1}{\sqrt{2 \pi}} \int_{-\infty}^{\infty} \cos \left(\frac{t}{q v}\right) \frac{q^{\operatorname{Sin}(v)}}{v} d v \\
& =\frac{-1}{\sqrt{2 \pi}} \int_{-\infty}^{\infty} \cos \left(\frac{t}{q v}\right) j_{0}(q ; v) d v
\end{aligned}
$$

exhibits wavelet properties, since its 0 th moment vanishes. A change of variables $v=1 /[q \omega]$ was made to obtain the last two equalities and express the solution in terms of the 0th order $q$-advanced spherical Bessel function.

\section{Conflict of Interests}

The authors declare that there is no conflict of interests regarding the publication of this paper.

\section{References}

[1] G. F. Carrier and C. E. Pearson, Partial Differential Equations: Theory and Technique, Academic Press, New York, NY, USA, 1976.

[2] G. B. Arfken and H. J. Weber, Mathematical Methods for Physicists, Elsevier, 5th edition, 2001.

[3] M. Reed and B. Simon, Scattering Theory III, Academic Press, New York, NY, USA, 1975.

[4] D. Pravica, N. Randriampiry, and M. Spurr, "On q-advanced spherical Bessel functions of the first kind and perturbations of the Haar Wavelet," 2014.

[5] D. W. Pravica, N. Randriampiry, and M. J. Spurr, "Reproducing kernel bounds for an advanced wavelet frame via the theta function," Applied and Computational Harmonic Analysis, vol. 33, no. 1, pp. 79-108, 2012.

[6] D. W. Pravica, N. Randriampiry, and M. J. Spurr, " $q$-advanced models for tsunami and rogue waves," Abstract and Applied Analysis, vol. 2012, Article ID 414060, 26 pages, 2012.

[7] I. Daubechies, Ten Lectures on Wavelets, vol. 61 of CBMSNSF Regional Coference Series in Applied Mathematics, SIAM, Philadelphia, Pa, USA, 1992.

[8] A. Lastra, S. Malek, and J. Sanz, "On q-asymptotics for linear $q$-difference-differential equations with Fuchsian and irregular singularities," Journal of Differential Equations, vol. 252, no. 10, pp. 5185-5216, 2012.

[9] A. Lastra and S. Malek, "On $q$-Gevrey asymptotics for singularly perturbed $q$-difference-differential problems with an irregular singularity," Abstract and Applied Analysis, vol. 2012, Article ID 860716, 35 pages, 2012.

[10] A. Lastra and S. Malek, "On parametric Gevrey asymptotics for singularly perturbed partial differential equations with delays," Abstract and Applied Analysis, vol. 2013, Article ID 723040, 18 pages, 2013.

[11] S. Malek, "On complex singularity analysis for linear partial $q$-difference-differential equations using nonlinear differential equations," Journal of Dynamical and Control Systems, vol. 19, no. 1, pp. 69-93, 2013.

[12] C. Zhang, "Analytic continuation of solutions of the pantograph equation by means of $\theta$-modular formula," http://arxiv.org/ abs/1202.0423. 
[13] O. Christensen, An Introduction to Frames and Riesz Bases, Applied and Numerical Harmonic Analysis, Birkhäuser, Boston, Mass, USA, 2003.

[14] M. Abramowitz and I. A. Stegun, Handbook of Mathematical Functions with Formulas, Graphs, and Mathematical Tables, vol. 55 of Applied Mathematics Series, National Bureau of Standards, Washington, DC, USA, 1964. 


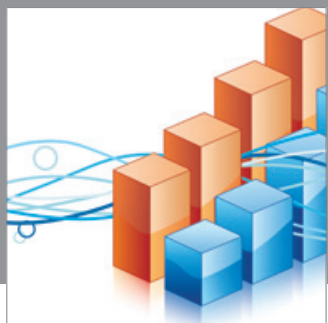

Advances in

Operations Research

mansans

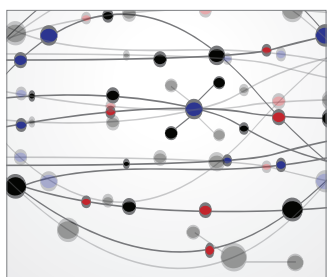

The Scientific World Journal
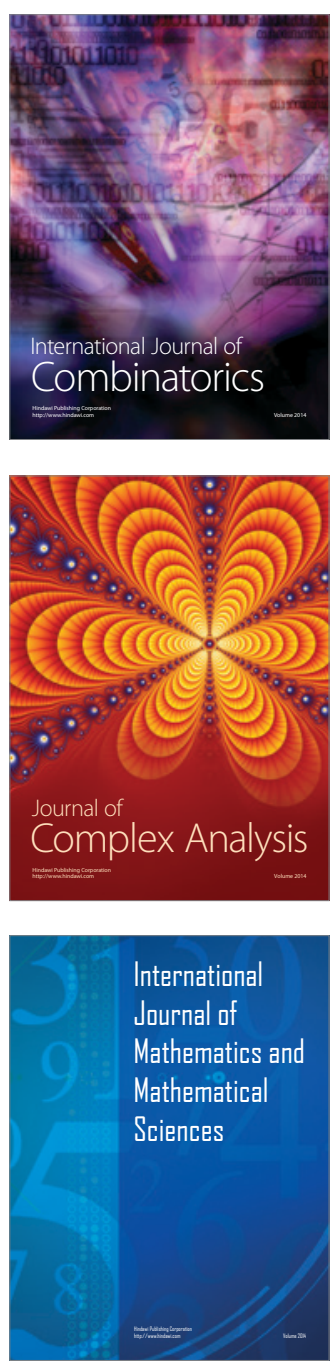
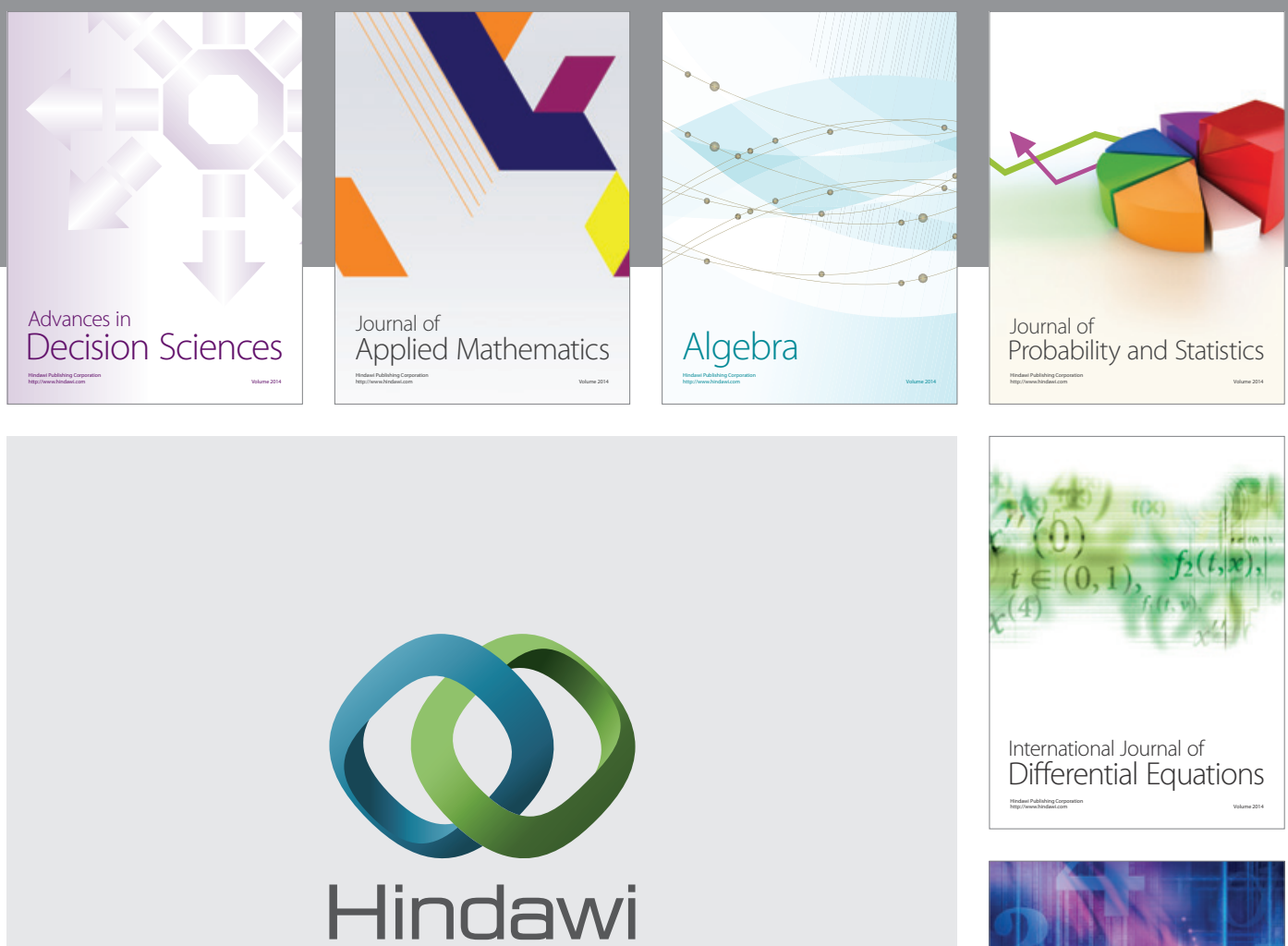

Submit your manuscripts at http://www.hindawi.com
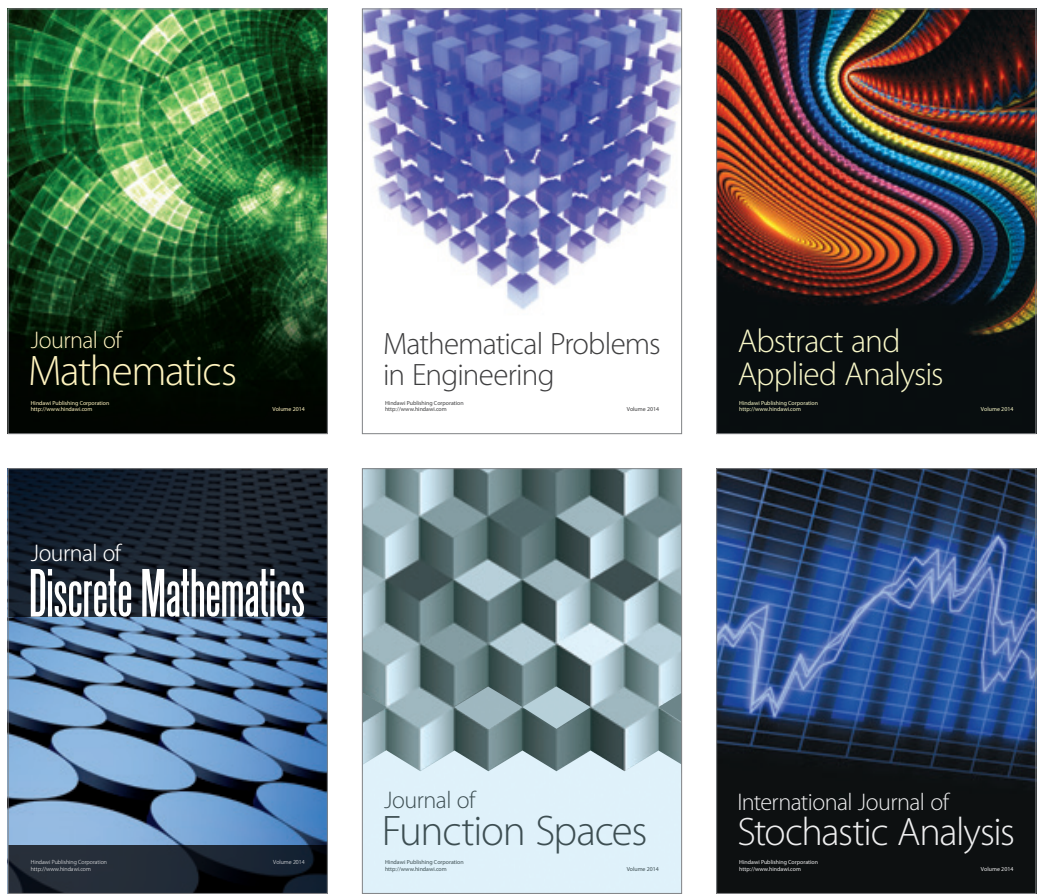

Journal of

Function Spaces

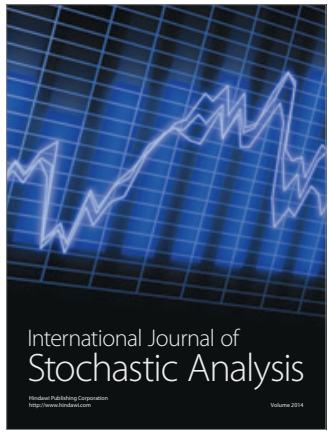

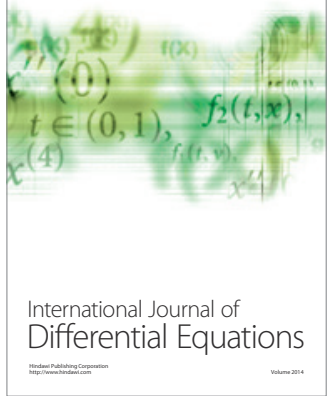
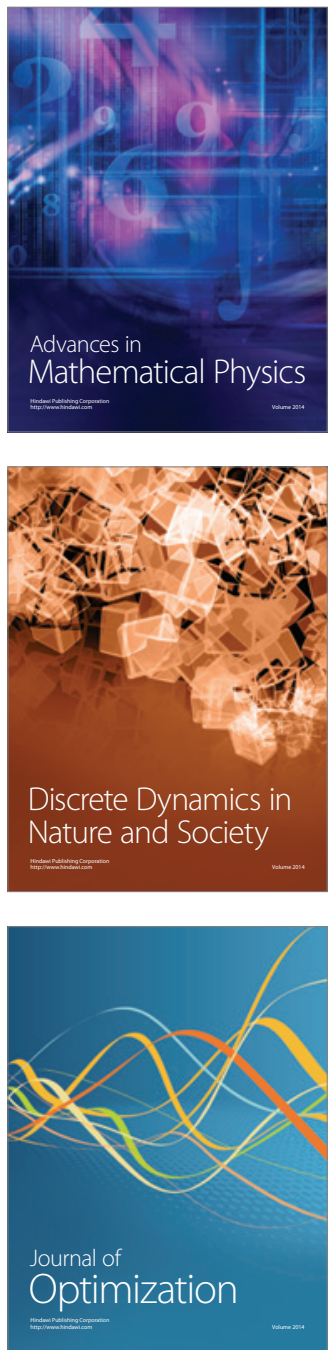